\title{
A Robust Collision Perception Visual Neural Network with Specific Selectivity to Darker Objects
}

\author{
Qinbing Fu, Member, IEEE, Cheng Hu, Jigen Peng, F. Claire Rind and Shigang Yue, Senior Member, IEEE
}

\begin{abstract}
Building an efficient and reliable collision perception visual system is a challenging problem for future robots and autonomous vehicles. The biological visual neural networks, which have evolved over millions of years in nature, and are working perfectly in the real world, could be ideal models for designing artificial vision systems. In the locust's visual pathways, a lobula giant movement detector, i.e. the LGMD2, has been identified as a looming perception neuron that responds most strongly to darker approaching objects relative to their backgrounds, similar situations which many ground vehicles and robots are often facing with. However, little has been done on modelling the LGMD2 and investigating its potential in robotics and vehicles. In this research, we build an LGMD2 visual neural network which possesses the similar collision selectivity of an LGMD2 neuron in locust, via the modelling of biased-ON and OFF pathways splitting visual signals into parallel ON/OFF channels. With stronger-inhibition (bias) in the ON pathway, this model responds selectively to darker looming objects. The proposed model has been tested systematically with a range of stimuli including real-world scenarios. It has also been implemented in a micro mobile robot and tested with real-time experiments. The experimental results have verified the effectiveness and robustness of the proposed model for detecting darker looming objects against various dynamic and cluttered backgrounds.
\end{abstract}

Index Terms-LGMD2, neuron model, collision perception, darker objects selectivity, $\mathrm{ON}$ and $\mathrm{OFF}$ pathways, mobile robots

\section{INTRODUCTION}

$\mathbf{C}$ OLLISIONS happen at every second in the real world, which often result in serious accidents and fatalities. In the future, every kind of artificial mobile machines, such as ground vehicles, robots, unmanned aerial vehicles (UAVs), should have good capability to detect and avoid collisions. However, current approaches for collision detection like radar, laser, infra-red, ultrasound, vision sensors or combinations of these are far from acceptable level in terms of reliability, energy consumption, price and size. A new type of reliable, low-cost, energy-efficient and miniaturised collision detection sensors is required to make future autonomous mobile machines safe to serve human society.

This research has been supported by the EU Horizon 2020 projects STEP2DYNA (691154) and ULTRACEPT (778062). Corresponding author: Shigang Yue (syue@lincoln.ac.uk). Qinbing Fu (qifu@lincoln.ac.uk)

Q. Fu, C. Hu and S. Yue are with the Machine Life and Intelligence Research Centre, School of Mechanical and Electrical Engineering, Guangzhou University, Guangzhou 510006, China, and also with the Computational Intelligence Lab/Lincoln Centre for Autonomous Systems, School of Computer Science, University of Lincoln, Lincoln LN6 7TS, United Kingdom.

J. Peng is with the School of Mathematics and Information Science, Guangzhou University, Guangzhou 510006, China.

F. C. Rind is with the Institute of Neuroscience/Centre for Behaviour and Evolution, Newcastle University, Newcastle NE1 7RU, United Kingdom.
In nature, insects, though with tiny brains, possess almost perfect sensory systems for timely collision sensing and avoidance within dynamic environments. As examples of visually guided navigation, it was discovered early on that locusts can see in light levels equivalent to starlight during migratory flights [1], and fly in swarms for hundreds of miles free of collision [2]. Nocturnal insects successfully forage in the forest at night without collision [3], [4]. The underlying mechanisms in biological visual pathways are prominent and powerful model systems to build collision-detecting systems, as reviewed in [5]-[8].

Specifically, a group of lobula giant movement detectors (LGMDs) in the locust's visual system has been discovered sensitive to looming objects [9]-[15]. The LGMD1 was first identified as a movement detector and gradually recognised as a looming objects detector, e.g. [11], [15]. In the same lobula layer, the LGMD2 was identified as a darker looming objects detector with unique characters that are different to the LGMD1, i.e. the LGMD2 lacks a ventrally located feedforward inhibitory (FFI) pathway which conveys object-size dependent inhibition to directly suppress the neuron [12], as illustrated in Fig. 1. Though both giant neurons are physically close to each other and have similar pre-synaptic structures, the LGMD2 possesses different selectivity to only darker objects moving in depth relative to their backgrounds [12], [14].

Computationally modelling such fascinating looming sensitive neurons will not only deepen our understanding of the very complex biological visual processing, but also shed light on building robust collision perception visual systems for robots and vehicles. In the past decade, the LGMD1 neuron has been modelled with a good number of studies and tested in ground robots, e.g. [16]-[19], and recently in UAVs [20], [21]. These LGMD1-based modelling studies have demonstrated that the biological visual systems can be good paradigms to develop energy-efficient and reliable collision detection visual systems for real-world applications.

Compared to the LGMD1, the LGMD2 neuron only detects darker objects that approach within bright background rather than any other categories of visual movements. Realising this feature will undoubtedly enhance the selectivity to collisions caused by darker approaching objects. However, very little LGMD2 modelling work has been done to demonstrate its collision-detecting ability and potential in real-world applications, due to two main aspects of difficulties:

1) On the aspect of biology, the LGMD2's neural circuitry still remains largely unknown compared to the LGMD1, including both its pre-synaptic structure and post-synaptic target neuron [15]. Therefore, understanding the LGMD2's 


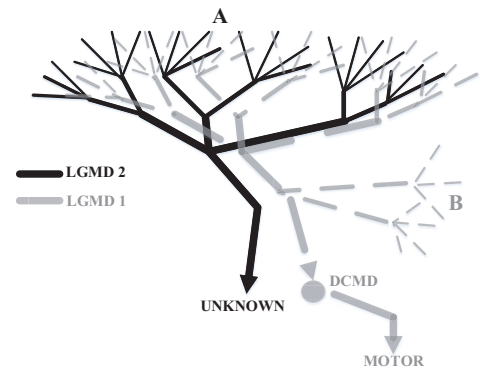

Fig. 1. Schematic diagram of the LGMDs morphology. Sub-field A indicates the pre-synaptic dendritic structures of both LGMDs. Sub-field B indicates the FFI pathway of LGMD1 which is absent from LGMD2 [12]. DCMD is a one-to-one post-synaptic target neuron to LGMD1 conveying neural signals to motor; the partner neuron of LGMD2 remains unknown [12], [15].

underlying neural mechanisms forming its specific selectivity to only darker looming objects is difficult.

2) Regarding computational modelling, implementing the LGMD2's specific collision selectivity to only darker objects has always been a challenging problem. Until recent years, the research progress made by biologists on biological ON and OFF pathways for motion perception [22], [23] has promoted the proposed LGMD2 modelling study with speculations that such dual-pathways exist also in the LGMD2's pre-synaptic circuit (see sub-field A in Fig. 1) to fulfil its specific characteristics.

Building upon our early partial studies on the LGMD2 [24][26], this article investigates the LGMD2's unique features through systematic modelling and experimental research, and demonstrates its potential as a robust collision perception visual system for mobile robots. The main contributions of this paper can be summarised as follows:

- This paper proposes a first systematic modelling study of the LGMD2 in the locust's visual system. For the first time, the LGMD2's specific characteristics and selectivity to only darker objects has been realised by the proposed visual neural network.

- The proposed modelling of ON and OFF pathways can implement both the LGMD1 [17] and the proposed LGMD2 which evidences that such structures and mechanisms play roles in the locust's visual system, though little physiological and anatomical evidence has been found to date.

- We develop a novel adaptive inhibition mechanism that works effectively to adjust local lateral inhibitions for shaping the LGMD2's unique selectivity at some critical moments by rapid or large-field movements. The model produces similar response to the locust LGMD2 [12].

- This research yields a simple and effective vision-based collision detection solution for mobile machines like robots that requires only an ordinary camera system and few computational resources.

The rest of this article is organised as follows: Section II reviews relevant fields of studies. Section III introduces the proposed LGMD2 visual neural network. Section IV presents systematic experiments and results. Further discussion is given in Section V. Section VI concludes this research.

\section{RELATED WORK}

Within this section, we briefly review related works in the areas of 1) conventional computer vision techniques and 2) bio-inspired methods for collision detection, 3) neural properties of the LGMD2, notably the differences to the LGMD1, 4) $\mathrm{ON}$ and OFF pathways in both biological and artificial vision systems.

\section{A. Computer Vision for Collision Detection}

For real-time collision detection, the vast majority of computer vision methods apply object-scene segmentation, estimation, localisation or classification based algorithms [27], [28]. Some collision detectors have been used in ground vehicles [27] and UAVs [29] for the purpose of improving navigation safety. As emergence of new types of visual sensors like RGBD, e.g. [30], [31] and event-driven cameras, e.g. [32], [33], the collision detection strategies can be enriched with more abundant visual features extracted for implementing obstacle recognition, object segmentation and map construction etc. More specifically, compared to standard cameras, these new visual sensors have a very high dynamic range that guarantees good performance to detect high-speed motions [33].

However, the conventional computer vision techniques for collision detection are either computationally costly or heavily reliant on specialised visual sensors. In addition, the efficiency and performance of these approaches also depend on the complexity of real physical scenes. Therefore, a new type of miniaturised, low-cost, low-power and reliable visual collision detectors is demanded for future intelligent machines interacting within complex dynamic environments.

\section{B. Biologically inspired Visual Collision Detectors}

Millions of years of evolutionary development has endowed, in nature, animals with robust and efficient collision-detecting visual systems. As outstanding examples, flying insects that demonstrate amazing collision perception and avoidance abilities, have been researched with considerable biological and modelling studies [5]-[8]. More concretely, a significant number of models come from optic flow (OF)-based strategies in the flying insects' visual systems [8]. The OF-based method has been successfully applied to a variety of flying robots for guiding insect-like behaviours including collision avoidance in flight [5], [7], [34], [35]. Such a strategy mimics the functionality of bilateral compound eyes of flying insects at ommatidia level. The local apparent motion flows containing direction and intensity information are captured and computed by 'delay-and-correlation' algorithms [5], [8], [22]. A field of local motion vectors is thus formed. However, to the best of our knowledge, a limitation exists that it is mainly used for sensing lateral rather than frontal collision threats.

On the other hand, the giant neurons like the LGMD1 in the locust's visual systems respond most strongly to frontal looming objects over other kinds of movements [11], [15], [36], [37]. As a powerful model system, the LGMD1 has been built as a quick collision detector and applied to ground vehicle scenarios, e.g. [38]-[40], mobile robots, e.g. [16]-[18], [41], UAVs [20], [21], and also embodied in hardware implementation like the FPGA [42]. Compared to the OF-based approaches, the LGMD1 models detect potential collisions by reacting to expanding edges of objects that approach. With 
similar ideas, Yue and Rind computationally modelled another group of directionally selective neurons (DSNs) in locusts for collision detection [43]. Compared with the LGMD1based models, the DSNs visual neural networks can provide additional edge-direction information of looming objects.

In general, most of these bio-inspired systems have been used to guide mobile robots in navigation. They nevertheless work individually; integrating different methods in the future could further enhance robots' obstacle avoidance capabilities.

\section{Characterisation of the LGMD2}

In comparison with the LGMD1, few biological [12], [14], [15] and modelling [24]-[26] studies have touched upon the LGMD2 due to the difficulties mentioned in the last section. Similarly to the LGMD1, the LGMD2 responds selectively to looming objects, with increasing firing rates peaked before the objects reaching a particular angular size in the field of view [12]. It is rigorously inhibited during either the whole-field luminance change or grating movements [12]. Moreover, when challenged by translation with constant intensity, the LGMD2 is excited for a short while then inhibited very soon, early before the end of translation [12] (see Fig. 4 in Section IV).

Not limited to that, biologists have recognised the LGMD2's unique looming selectivity. A notable feature of the LGMD2 is that it is only sensitive to darker approaching objects against bright background, while not able to detect brighter or white objects approaching within dark background [12]. Furthermore, biologists have also realised that the LGMD2 has preference for the light-to-dark luminance transitions (or OFF contrast). For example, only the direction of movement of a single dark edge advancing rightward or a single light edge retracting with OFF contrast excites the LGMD2 [12] (see Fig. 4 in Section IV). In contrary, the LGMD1 responds to both the dark-to-light luminance change (or ON contrast) and OFF contrast [17]. The LGMD2's characteristics make it outside of normal expectation and an unique neuron to model.

\section{ON and OFF Pathways for Motion Perception}

The proposed LGMD2 visual neural network is featured by a new bio-plausible structure of the $\mathrm{ON}$ and OFF pathways which separates the visual processing from the photoreceptor layer into parallel computation. The ON and OFF pathways have been discovered in the preliminary visual systems of many animal species such as insects like flies [22], [44], and vertebrates [45] including rabbits [22], mice [23], cats [46] and monkeys [47]. Such a structure reveals an essential principle of biological visual processing, that is, the motion information is separated into parallel $\mathrm{ON}$ and OFF channels encoding brightness increments $(\mathrm{ON})$ and decrements $(\mathrm{OFF})$, separately [22], [23], [48]. For locusts, there is very limited evidence showing or suggesting the existence of such polarity mechanisms [10], [13], [15], [49].

With regard to locust LGMDs based modelling studies, a seminal LGMD1 work applied similar ON and OFF mechanisms for collision detection in real-world scenarios [50]. Recent researches also demonstrated the effectiveness of such a novel structure to implement a biological LGMD1 [17], [40].

\section{Formulation of the Visual Neural Network}

Within this section, the proposed method will be presented in detail. We first introduce the core structures of the LGMD2 visual neural network, then elaborate on its components in the following subsections.

To achieve the LGMD2's specific selectivity to darker objects, we highlight the modelling of 1) biased-ON and OFF pathways, and 2) adaptive inhibition mechanism. Generally speaking, the proposed visual neural network consists of five layers, including photoreceptor $(\mathbf{P})$, excitation $(\mathbf{E})$, inhibition (I), summation (S), grouping (G) layers and an LGMD2 cell. The E, I and S layers are embodied in the parallel ON and OFF pathways. As illustrated in Fig. 2, the luminance change at local pixel level is captured by the $\mathrm{P}$ layer; the luminance increments flow into ON channels; whilst the decrements flow into OFF channels; each polarity pathway depicts spatiotemporal neural computation between local excitations and inhibitions; notably, the stronger inhibition (namely 'bias') is put forth in the ON pathway to achieve the LGMD2's unique selectivity to darker objects moving in depth; all the presynaptic local excitations reaching the LGMD2 are integrated to form the membrane potential which is then shaped by a spike frequency adaptation (SFA) mechanism and generating spikes to indicate potential collision threats by darker objects. Moreover, the adaptive inhibition mechanism is accomplished by a photoreceptors mediation (PM) pathway to adjust local biases on lateral inhibitions in either ON/OFF pathway.

Our motivation to introduce the two core structures in this modelling research is mainly based on the following points:

- The ON and OFF pathways work effectively to separate different collision selectivity between the LGMD1 and the LGMD2, and can implement functions of both the LGMDs. With speculations that the ON channels are rigorously sieved by stronger lateral inhibitions, the specific selectivity of LGMD2 can be separated from the LGMD1. As a result, such a structure could be used to construct a general model for the locust's looming sensitive giant neurons.

- The biological LGMD2 lacks the FFI pathway in the LGMD1's circuitry (see sub-field B in Fig. 1), but represents similar inhibitions to the LGMD1 on rapid or large-field luminance change. To achieve this, the proposed LGMD2 model is characterised by a new adaptive inhibition mechanism to adjust local lateral inhibitions in both polarity pathways adapting to various visual movements.

\section{A. Photoreceptors Layer}

The first computational layer consists of photoreceptors arranged in a matrix, which captures grey-scale luminance and computes temporal derivative of every pixel to get motion information [11], [17], [51]. Let $L(x, y, t) \in \mathbb{R}^{3}$ denote the input image streams, where $x, y$ and $t$ are spatial and temporal positions, respectively. The computation can be defined as

$$
\begin{gathered}
P(x, y, t)=L(x, y, t)-L(x, y, t-1)+\sum_{i=1}^{n_{p}} a_{i} \cdot P(x, y, t-i), \\
a_{i}=\left(1+e^{i}\right)^{-1},
\end{gathered}
$$




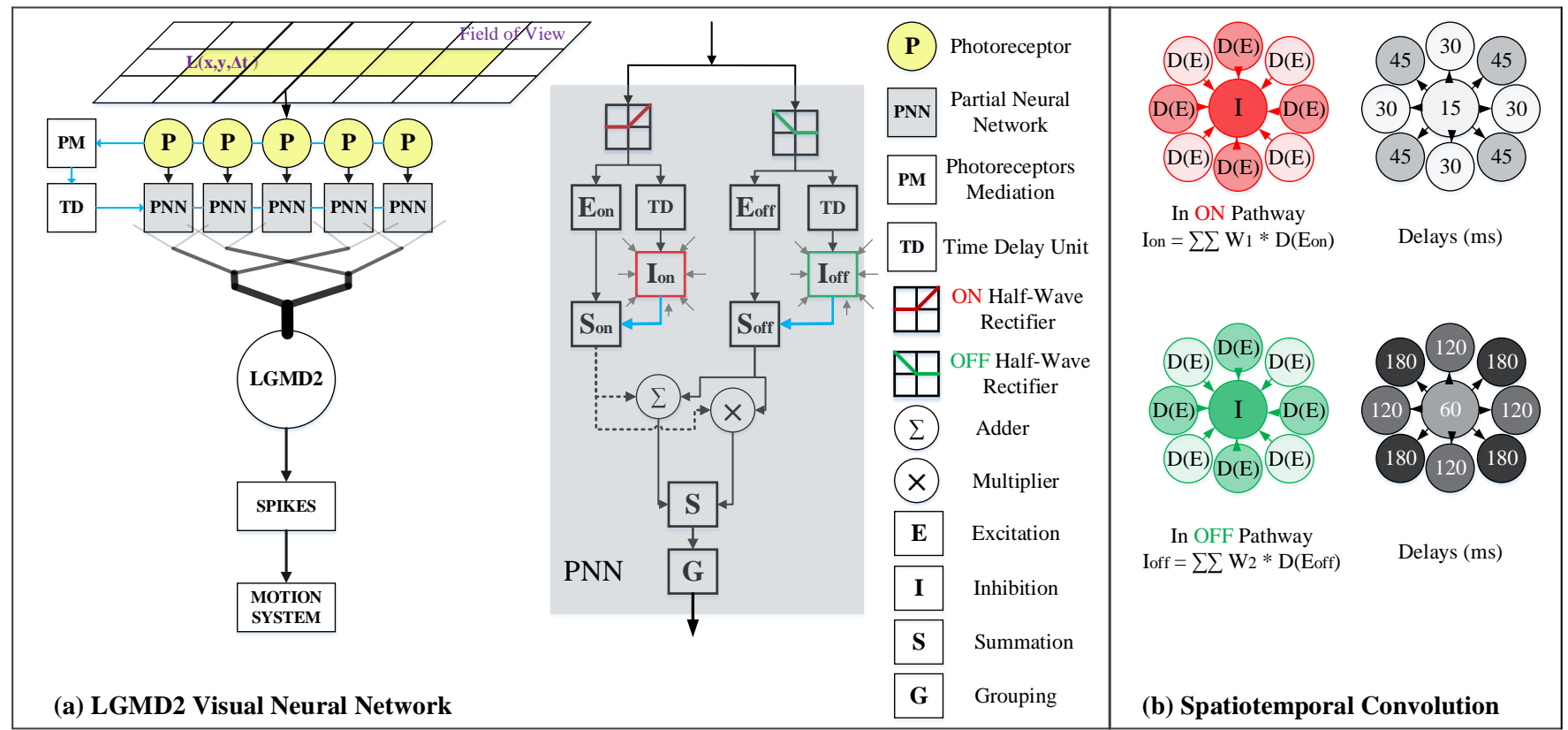

Fig. 2. Schematic of the LGMD2 visual neural network and spatiotemporal convolution. For clear illustration, only five photoreceptors and corresponding downstream processing are depicted. Each photoreceptor captures luminance (L) change at local pixel level within the field of view. Each connects with a partial neural network, i.e. ON and OFF channels. The LGMD2 cell integrates the whole pre-synaptic local excitations. A separate photoreceptors mediation (PM) pathway (blue lines) adjusts the local inhibitory strength in either ON/OFF channel, at every time step. The local ON/OFF inhibitions are generated by convolving surrounding delayed ON/OFF excitations. Stronger inhibitions are formed in all the ON channels to sieve the ON excitations (dashed lines).

where $P(x, y, t)$ denotes luminance change of pixel $(x, y)$ with respect to time $t$. The persistence of luminance change could last for a short while of $n_{p}$ number of frames [51], and $a_{i}$ stands for a decay coefficient.

\section{B. $O N$ and $O F F$ Mechanisms}

After that, motion information is passed into separated ON/OFF channels, i.e., the start of visual processing in the ON and OFF pathways. The functions of ON and OFF transient cells are implemented by half-wave rectifying. More precisely, for each photoreceptor, the luminance increment flows into the ON channel, whilst the brightness decrement flows into the OFF channel. That is,

$$
\begin{aligned}
& P_{o n}(x, y, t)=[P(x, y, t)]^{+}+\alpha_{1} \cdot P_{o n}(x, y, t-1), \\
& P_{o f f}(x, y, t)=-[P(x, y, t)]^{-}+\alpha_{1} \cdot P_{o f f}(x, y, t-1) .
\end{aligned}
$$

Here, $[x]^{+}$and $[x]^{-}$denote $\max (0, x)$ and $\min (x, 0)$, respectively. A small fraction $\left(\alpha_{1}\right)$ of residual signals is allowed to pass through. Such mechanisms have also demonstrated the efficacy to encode other insect-inspired motion detectors including small target movement detectors of the flying insects [52], [53] and angular velocity detectors of the bee [54], [55] and direction selective neurons of the Drosophila [56], [57].

\section{Neural Computation in $O N$ and OFF Pathways}

In the previous LGMD1 based models, e.g. [11], [17], [41], [51], there are two kinds of signal flows, i.e. the excitation and the inhibition competing with each other. If the former one wins, the neuron is activated to spike; otherwise, the neuron remains quiet. More precisely, the lateral inhibitions are time delayed which cut down the motion-dependent excitations with an object growing on the visual field. Such a competition plays crucial roles of shaping the looming selectivity of the locust's giant neurons. For modelling the LGMD2, we apply similar strategies: each pathway depicts the competition between local polarity excitations and inhibitions. For implementing the LGMD2's specific selectivity, the stronger inhibitions are put forth in all the ON channels forming a biased-ON pathway.

1) Competition in the ON Pathway: In the ON pathway, the local excitation (E) reaches the $E_{\text {on }}$ unit without temporal latency; meanwhile, it is fed into a time delay unit (TD). The local inhibition (I) in the $I_{o n}$ unit is thus formed by convolving surrounding delayed local excitations $\hat{E}_{o n}$ (see $D\left(E_{o n}\right)$ in Fig. 2(b)). The whole process can be defined as

$$
\begin{gathered}
E_{o n}(x, y, t)=P_{o n}(x, y, t) \\
\hat{E}_{o n}(x, y, t)=\alpha_{2} E_{o n}(x, y, t)+\left(1-\alpha_{2}\right) E_{o n}(x, y, t-1) \\
\alpha_{2}=\tau_{i n} /\left(\tau_{1}+\tau_{i n}\right), \\
I_{o n}(x, y, t)=\sum_{i=-1}^{1} \sum_{j=-1}^{1} \hat{E}_{o n}(x+i, y+j, t) W_{1}(i+1, j+1) .
\end{gathered}
$$

$\tau_{1}$ and $\tau_{i n}$ are two time constants in milliseconds, wherein $\tau_{1}$ stands for the excitation delay time (see Fig. 2(b)) and $\tau_{i n}$ is the time interval between consecutive frames of digital signals. $W_{1}$ stands for a convolution kernel that meets the following matrix:

$$
W_{1}=\left(\begin{array}{ccc}
1 / 4 & 1 / 2 & 1 / 4 \\
1 / 2 & 2 & 1 / 2 \\
1 / 4 & 1 / 2 & 1 / 4
\end{array}\right) .
$$

In the convolution process, the centre cell has the highest weighting and the shortest delay; the four nearest cells have the moderate weighting and delay; the four diagonal cells share the lowest weighting and longest delay (see Fig. 2(b)). The selection of spatiotemporal parameters originates from the biological research on the LGMD [15]: the delayed signal 
flows spread out to their surrounding area to form lateral inhibitions affecting and cutting down the motion-dependent excitations.

2) Competition in the OFF Pathway: In the OFF pathway, signal flows conveyed by the OFF cells form the local excitations to the $E_{\text {off }}$ unit without latency, and the delayed local inhibitions $\hat{E}_{\text {off }}$ (see $D\left(E_{\text {off }}\right)$ in Fig. 2(b)) in the $I_{o f f}$ unit. These processes are defined as

$$
\begin{gathered}
E_{o f f}(x, y, t)=P_{o f f}(x, y, t), \\
\hat{E}_{o f f}(x, y, t)=\alpha_{3} E_{o f f}(x, y, t)+\left(1-\alpha_{3}\right) E_{o f f}(x, y, t-1), \\
\alpha_{3}=\tau_{i n} /\left(\tau_{2}+\tau_{i n}\right), \\
I_{o f f}(x, y, t)=\sum_{i=-1}^{1} \sum_{j=-1}^{1} \hat{E}_{o f f}(x+i, y+j, t) W_{2}(i+1, j+1) .
\end{gathered}
$$

Compared with the ON channels, the delay time constant $\tau_{2}$ at each local cell is larger (see Fig. 2(b)): prolonging the delay will reduce the local inhibitions. $W_{2}$ fits the following matrix with lower weightings:

$$
W_{2}=\left(\begin{array}{ccc}
1 / 8 & 1 / 4 & 1 / 8 \\
1 / 4 & 1 & 1 / 4 \\
1 / 8 & 1 / 4 & 1 / 8
\end{array}\right)
$$

Following the generation of local ON/OFF excitations and inhibitions, there are local ON/OFF summation (S) cells in both channels depicting a purely linear computation. That is,

$$
\begin{aligned}
S_{o n}(x, y, t) & =\left[E_{o n}(x, y, t)-w_{1}(t) \cdot I_{o n}(x, y, t)\right]^{+}, \\
S_{o f f}(x, y, t) & =\left[E_{o f f}(x, y, t)-w_{2}(t) \cdot I_{o f f}(x, y, t)\right]^{+},
\end{aligned}
$$

where $w_{1}(t)$ and $w_{2}(t)$ are time-varying local biases to control the intensity of inhibitory flows.

\section{Adaptive Inhibition Mechanism}

As introduced in Sections I and II, the LGMD2 circuitry lacks the FFI pathway which can directly suppress the neuron if luminance changes rapidly over a large area in the field of view. However, the LGMD2 also shows similar vigorous inhibition in the physiological experiments [12], at some critical moments of either the end of approach by darker objects or the start of recession by brighter objects. To fulfil this character, we propose the original modelling of adaptive inhibition mechanism to adjust the $\mathrm{ON}$ and $\mathrm{OFF}$ time-varying biases in Eq. 14. As illustrated in Fig. 2, this is implemented by a PM pathway with a slight delay, which is given by

$$
P M(t)=\sum_{x=1}^{R} \sum_{y=1}^{C}|P(x, y, t)| \cdot(C \cdot R)^{-1},
$$

$\hat{P M}(t)=\alpha_{4} P M(t)+\left(1-\alpha_{4}\right) P M(t-1), \alpha_{4}=\tau_{\text {in }} /\left(\tau_{3}+\tau_{\text {in }}\right)$,

$w_{1}(t)=\max \left(w_{3}, \frac{\hat{P M}(t)}{T_{p m}}\right), w_{2}(t)=\max \left(w_{4}, \frac{\hat{P M}(t)}{T_{p m}}\right)$.

$C$ and $R$ denote the columns and rows of the photoreceptors layer; $\tau_{3}$ stands for a delay time constant. $T_{p m}$ is a predefined

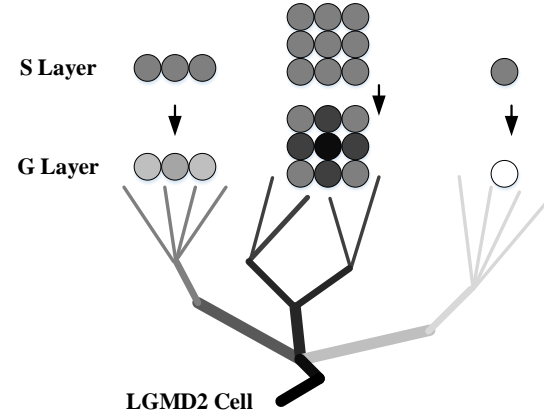

Fig. 3. Schematic illustration of $\mathrm{G}$ layer processing. The $\mathrm{S}$ cells surrounded by strong excitations gain bigger passing coefficients; the isolated ones get smaller passing coefficients and may be ruled out in the G layer by threshold. Circles represent excitations in $\mathrm{S}$ and $\mathrm{G}$ layers. The excitation strength is indicated by grey levels where black represents the strongest excitation.

threshold; $w_{3}$ and $w_{4}$ denote the different baselines of bias in $\mathrm{ON}$ and OFF pathways, respectively. In addition, this novel mechanism works effectively to enable the LGMD2 model to adapt to different levels of background complexity. More precisely, the local lateral inhibition gets stronger when luminance changes dramatically within the field of view. This mechanism well accounts for the explored physiological features of the LGMD2 [12], with which the giant neuron is inhibited by large-field movements such as gratings, rapid turning scenes, end of approach and start of recession etc. Performing in real physical world, this can also enhance the LGMD2's selectivity to darker objects that approach over other categories of movements against various dynamic backgrounds.

\section{E. Summation and Grouping Layers}

As exhibited in the PNN of Fig. 2, there are interactions between local excitations from the ON and OFF channels in the summation (S) unit. The calculation obeys a supra-linear rule as

$$
\begin{aligned}
S(x, y, t) & =\theta_{1} \cdot S_{o n}(x, y, t)+\theta_{2} \cdot S_{o f f}(x, y, t) \\
& +\theta_{3} \cdot S_{\text {on }}(x, y, t) \cdot S_{o f f}(x, y, t),
\end{aligned}
$$

where $\left\{\theta_{1}, \theta_{2}, \theta_{3}\right\}$ denote the combination of term coefficients that allows the $\mathrm{S}$ unit to represent different 'balances' between local polarity excitations and mediate influences by $\mathrm{ON}$ and OFF contrast. This method can realise either linear or multiplicative neural computation, which has demonstrated the effectiveness of implementing the small target movement detector [48] and also the LGMD1 [17]. For the proposed model, this can also play a role of enhancing the LGMD2's preference for OFF contrast.

Cascaded the $\mathrm{S}$ layer, the proposed neural network is featured by a grouping (G) layer (see Fig. 3), for the purpose of reducing isolated noise and improving the extraction of colliding objects against complex backgrounds with detail noise [51]. This is implemented with a passing coefficient matrix $\mathrm{Ce}$ obtained by a convolution process with an equalweighted kernel $W_{g}$. That is,

$$
\begin{gathered}
C e(x, y, t)=\sum_{i=-1}^{1} \sum_{j=-1}^{1} S(x+i, y+j, t) W_{g}(i+1, j+1), \\
W_{g}=\frac{1}{9} \times\left(\begin{array}{lll}
1 & 1 & 1 \\
1 & 1 & 1 \\
1 & 1 & 1
\end{array}\right)
\end{gathered}
$$




$$
\begin{aligned}
G(x, y, t) & =S(x, y, t) \cdot C e(x, y, t) \cdot \omega(t)^{-1}, \\
\omega(t) & =\max \left([C e]_{t}\right) \cdot C_{\omega}^{-1}+\Delta_{C} .
\end{aligned}
$$

$\omega$ is a scale parameter computed at every time step; $C_{\omega}$ is a constant; $\Delta_{C}$ stands for a small real number. Furthermore, the isolated and decayed excitations are filtered by

$$
\hat{G}(x, y, t)=\left\{\begin{array}{cl}
G(x, y, t), & \text { if } G(x, y, t) \cdot C_{d e} \geq T_{d e} \\
0, & \text { otherwise }
\end{array}\right.
$$

where $C_{d e}$ stands for the decay coefficient and $C_{d e} \in(0,1)$; $T_{d e}$ denotes the decay threshold. As a result, the grouped excitations in the $\mathrm{S}$ layer representing expanding edges become stronger when reaching the $\mathrm{G}$ layer and the background details caused isolated excitations are largely filtered out.

\section{F. LGMD2 Cell}

After the pre-synaptic visual processing, an LGMD2 cell integrates all local excitations from the $G$ layer to form the membrane potential as the following:

$k(t)=\sum_{x=1}^{R} \sum_{y=1}^{C} \hat{G}(x, y, t), K(t)=\left(1+e^{-k(t) \cdot\left(C \cdot R \cdot \alpha_{5}\right)^{-1}}\right)^{-1}$,

where $\alpha_{5}$ denotes a scale coefficient, and the output is regulated within $[0.5,1)$.

Subsequently, following our recent modelling studies on biological LGMDs [17], [25], [40], we apply an SFA mechanism to further sharpen up the LGMD2's selectivity, which is defined as

$$
\begin{gathered}
\hat{K}(t)=\left\{\begin{array}{l}
\alpha_{6}(\hat{K}(t-1)+K(t)-K(t-1)), \\
\text { if }(K(t)-K(t-1)) \leq T_{s f a} \\
\alpha_{6} K(t), \text { otherwise }
\end{array}\right. \\
\alpha_{6}=\tau_{4} /\left(\tau_{4}+\tau_{i n}\right),
\end{gathered}
$$

where $\alpha_{6}$ is a coefficient that indicates adaptation rate to visual movements; $T_{s f a}$ denotes a small real number as the threshold; $\tau_{4}$ is a delay time constant. Generally speaking, such a mechanism is a reduction of neuron firing rate to stimuli with constant or decreasing intensity, e.g. objects recede or translate at a constant speed; it has little effect on accelerating motion with increasing intensity like looming [17]. The SFA mechanism is sensitive to the motion intensity gradient and ideal for shaping the LGMD2's response to darker objects approaching rather than merely translating or brighter objects receding, which can be clearly seen from the testing results in Fig. 5, 6 and 7 in Section IV.

After that, the membrane potential is exponentially mapped to spikes by an integer-valued function. That is,

$$
S^{\text {spike }}(t)=\left[e^{\left(\alpha_{7} \cdot\left(\hat{K}(t)-T_{s p i}\right)\right)}\right]
$$

where $T_{s p i}$ indicates the spiking threshold and $\alpha_{7}$ is a scale parameter affecting the firing rate, i.e., increasing it will bring about higher spiking frequency. Finally, the following
TABLE I

Setting Parameters of The Proposed LGMD2 Model

\begin{tabular}{l|l|l}
\hline Parameter & Description & Value \\
\hline$n_{p}$ & luminance persistence in frames Eq. 1 & $0 \sim 2$ \\
$\alpha_{1}$ & coefficient in half-wave rectifying Eq. 3 & 0.1 \\
$\tau_{1}$ & latency in ON channels Fig.2 & $15 \sim 45$ \\
$\tau_{i n}$ & time interval of input digital signal & $30 \sim 50$ \\
$\tau_{2}$ & latency in OFF channels Fig.2 & $60 \sim 180$ \\
$w_{3}$ & bias baseline in ON channels & 1 \\
$w_{4}$ & bias baseline in OFF channels & 0.5 \\
$\tau_{3}$ & latency in PM pathway Eq. 16 & 90 \\
$T_{p m}$ & threshold in PM pathway Eq. 17 & 10 \\
$\left\{\theta_{1}, \theta_{2}, \theta_{3}\right\}$ & term coefficients in S layer Eq. 18 & $\{0.5,1,1\}$ \\
$C_{\omega}$ & constant to calculate $\omega$ in Eq. 21 & 4 \\
$\Delta_{C}$ & small real number in Eq. 22 & 0.01 \\
$C_{d e}$ & decay coefficient in G layer & 0.5 \\
$T_{d e}$ & decay threshold in G layer & 15 \\
$R, C$ & row, column of visual field in pixels & adaptable \\
$\alpha_{5}$ & coefficient in sigmoid function Eq. 24 & $0.5 \sim 1$ \\
$\tau_{4}$ & time constant in SFA Eq. 26 & $500 \sim 1000$ \\
$T_{s f a}$ & small threshold in SFA & 0.003 \\
$\alpha_{7}$ & scale coefficient in spiking Eq. 27 & 4 \\
$T_{s p i}$ & spiking threshold in Eq. 27 & $0.65 \sim 0.78$ \\
$n_{t s}$ & time window by discrete digital frames & $4 \sim 8$ \\
$n_{s p}$ & number of spikes within $n_{t s}$ & $6 \sim 8$ \\
\hline
\end{tabular}

formulation is used to indicate a potential collision threat in real time robot experiments:

$$
\operatorname{Col}(t)=\left\{\begin{array}{l}
\text { True, if } \sum_{i=t-n_{t s}}^{t} S^{\text {spike }}(i) \geq n_{s p} \\
\text { False, otherwise }
\end{array}\right.
$$

where $n_{s p}$ denotes the number of spikes in a specified time window constituted by $n_{t s}$ successive digital signal frames.

\section{G. Setting Model Parameters}

The parameters set-up is described in Table I. The proposed visual neural network is featured by a feed-forward structure. All the parameters are decided empirically with considerations and optimisations of the functionality of proposed biologically plausible pathways and mechanisms to implement a biological LGMD2 neuron, and moreover as an embedded vision system in a miniaturised mobile robot. There is currently no learning method involved for setting the parameters. However, these can be searched or learned in evolutionary computation, e.g. the genetic algorithms similarly to the related bio-inspired modelling studies [43], [58], since the search space of the proposed model is large and there are many parameters involved. As listed in Table I, the adaptable parameters including $C, R$ are decided by the physical property of input image sequences, i.e. the resolution. More precisely, in the experiments, the video clips are $600 \times 600$ and $432 \times 240$ for synthetic and real-world input stimuli, respectively; the robot's field of view is set at $99 \times 72$.

The proposed LGMD2 model has been set up in Visual Studio (Microsoft Corporation). Data analysis and visualisations have been implemented in Matlab (The MathWorks, Inc. Natick, USA). Both the source code of algorithms and the neural network layer outputs representing the signal processing in multiple layers or channels can be found at https://github.com/fuqinbing/LGMD2-open-source. 


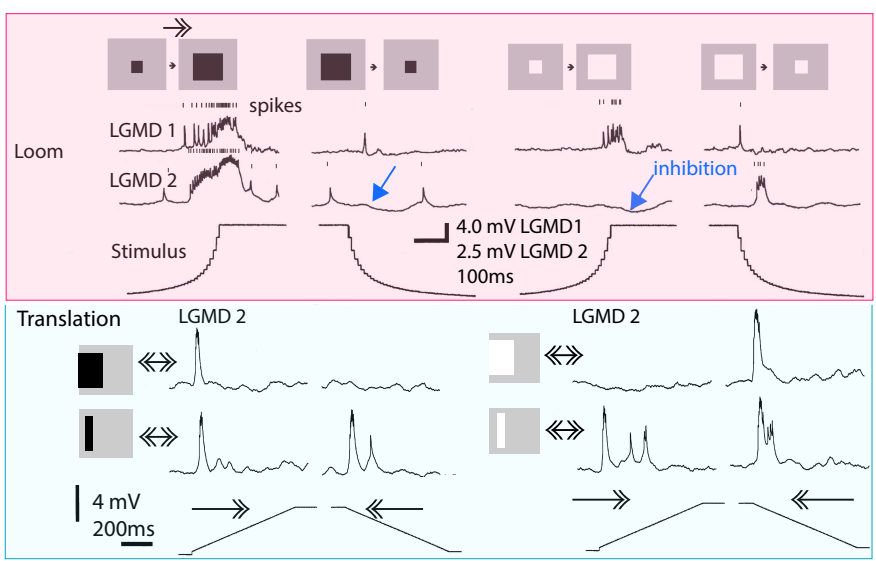

Fig. 4. Biological data on LGMD2 neural responses to darker and lighter objects looming and translating, adapted from [12]. The LGMD1 responds to all stimuli of looming, translating and elongating-shortening (a single edge translating); whilst the LGMD2 responds selectivity to darker object approaching, briefly to translation, lighter object receding, darker object elongating and lighter object shortening - the preference for OFF contrast.

\section{EXPERIMENTAL RESULTS AND ANALYSIS}

Within this section, systematic experiments are described which demonstrate how particular pathways and mechanisms contribute to the LGMD2's responses and selectivity. All the experiments can be categorised into two types of tests: the offline tests and the on-line tests. In the off-line tests, the input stimuli consist of synthetic and recorded image streams. We compare the model responses and selectivity with the previous biological data on LGMD2 [12] in Fig. 4. In the on-line tests, the proposed model is implemented in a miniaturised and vision-based mobile robot for real time experiments.

\section{A. Off-line Tests}

1) Synthetic Stimuli Testing: We start from testing the proposed model using computer-simulated movements. All the stimuli can be categorised into a few types including approach, recession, translation, elongation, shortening and sinusoidal grating following the physiological testing on LGMD2.

Firstly, we examine if the proposed model possesses similar unique selectivity like the biological LGMD2 as shown in Fig. 4. The results in Fig. 5 illustrate that the LGMD2 model responds selectively to darker looming object and is only shortly activated by recession of brighter object demonstrating the LGMD2's unique character. More precisely, when challenged against a darker approaching object, the LGMD2 releases membrane potential that rapidly increases as the image size projected in the field of view grows. However, the LGMD2 model shows no response to the darker object that recede. For a lighter (or white) looming object, the LGMD2 model remains quiet and is briefly activated during the start of recession. The proposed modelling of biased-ON and OFF pathways and SFA mechanism work effectively to achieve the required selectivity to darker objects with preference for only OFF contrast.

Secondly for the stimuli restricted to the X-Y plane, with darker or brighter object translations at constant speed, the LGMD2 model only shows a brief, weak response at the beginning of each course (see Fig. 6), which conforms to the biological research [12] (Fig. 4). Importantly, the LGMD2

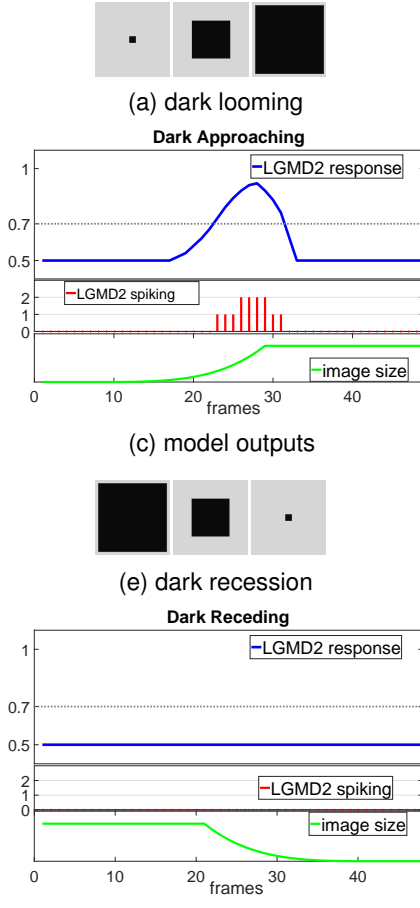

(g) model outputs

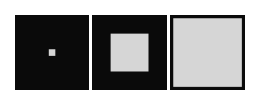

(b) bright looming

Light Approaching

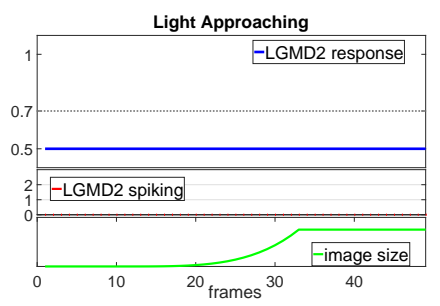

(d) model outputs

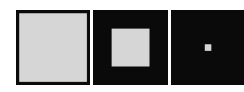

(f) bright recession Light Receding

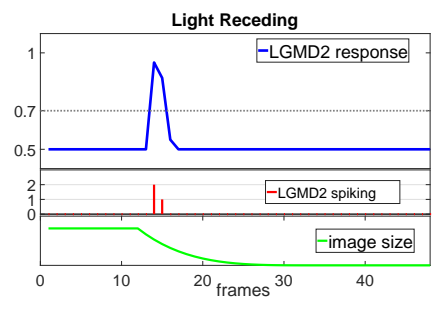

(h) model outputs
Fig. 5. The proposed LGMD2 model outputs by darker and brighter objects approaching and receding: membrane potential and spikes are depicted with change of image size shown at each bottom, the horizontal dashed line indicates firing threshold at 0.7 . The output at 0.5 indicates non-response.

responds to translations regardless of motion directions, which is different to the neural systems with direction selectivity to X-Y plane movements, e.g. [43], [52], [56]. As a special case of translating stimuli, the elongation and shortening movements represent the situations that objects translate very close to the field of view (Fig. 7). More precisely, the single translating edge leads to OFF contrast during dark-elongating and light-shortening; otherwise it gives rise to ON contrast. Similarly to the physiological results in Fig. 4, the proposed LGMD2 model responds selectively and briefly to the darkelongating and light-shortening stimuli with OFF contrast.

Finally in the synthetic stimuli tests, we test the LGMD2 model using sinusoidal grating movements with a wide range of spatial and temporal frequencies, which is a basic test for examining the robustness of biologically inspired visual systems performing against various dynamic and cluttered backgrounds [17]. Fig. 8 demonstrates that the proposed LGMD2 model is not responding to the grating stimuli with varied spatiotemporal frequencies at all. The proposed original modelling of adaptive inhibition mechanism plays an important role to mediate lateral inhibitions suppressing the neuron against gratings. The results indicate the proposed artificial neural network is robust to perform against a variety of dynamic and cluttered backgrounds.

2) Real Physical Stimuli Testing: After that, the proposed LGMD2 model is tested using recorded real-world visual stimuli including indoor scenes and outdoor ground vehicle scenarios. Notably, compared to the simulated scenes, there is much more background noise in the real physical world, noise such as light flash and shadows etc. In addition, unlike the simulated movements, the object's moving speeds could not be maintained at a constant level. Therefore, the visual 


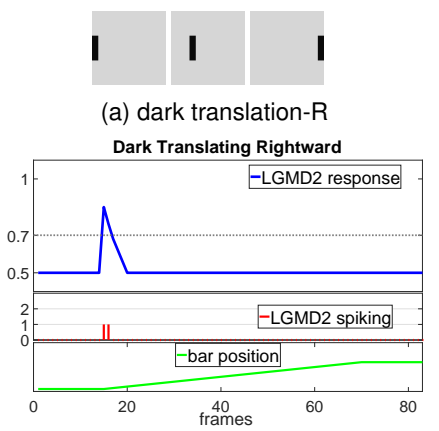

(c) model outputs
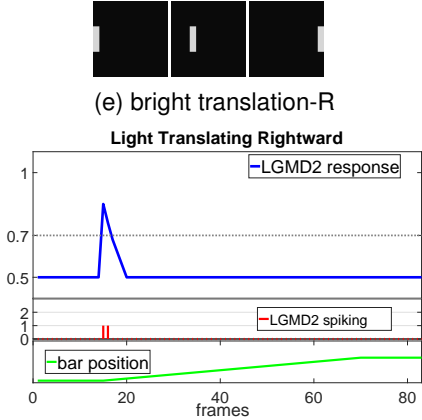

(g) model outputs (e) bright translation- $R$

\section{I I}

(b) dark translation-L

Dark Translating Leftward

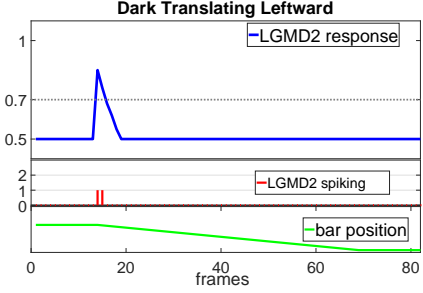

(d) model outputs

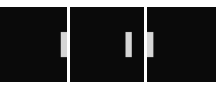

(f) bright translation-L Light Translating Leftward

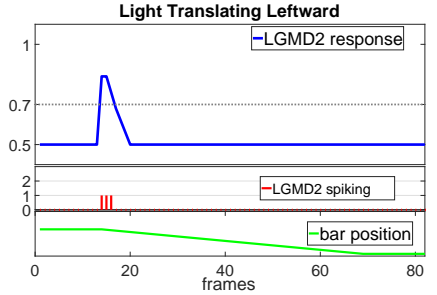

(h) model outputs
Fig. 6. The LGMD2 outputs by darker and brighter bars translating rightward $(\mathrm{R})$ and leftward $(\mathrm{L})$ with the change of bar position depicted at each bottom.

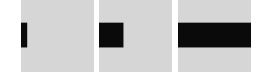

(a) dark elongation Dark Elongating

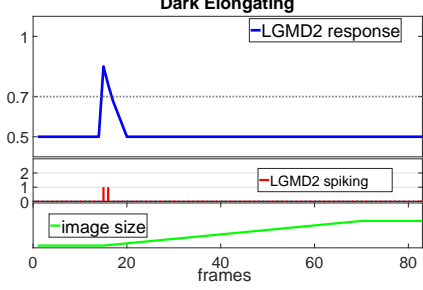

(c) model outputs

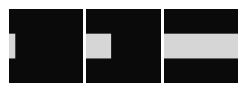

(e) bright elongation Light Elongating

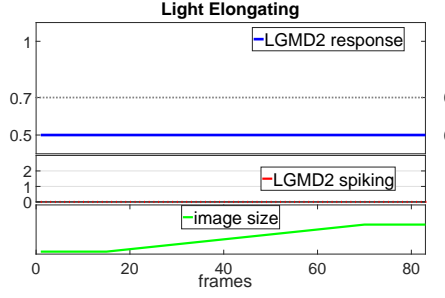

(g) model outputs

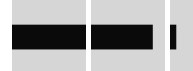

(b) dark shortening Dark Shortening

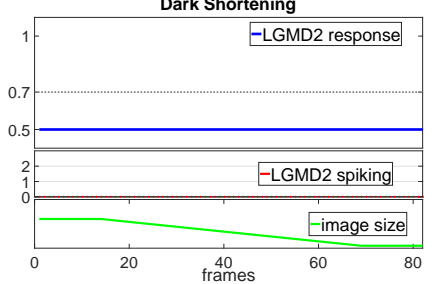

(d) model outputs

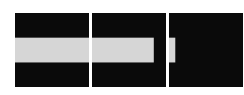

(f) bright shortening Light Shortening

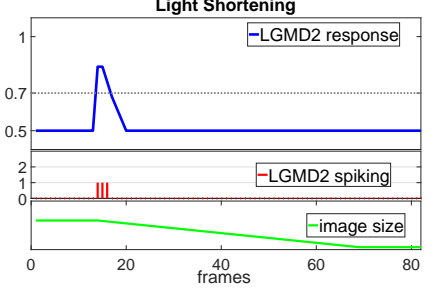

(h) model outputs
Fig. 7. The LGMD2 outputs by dark and light elongation and shortening movements with the change of image size depicted at each bottom.

challenges presented to the model are 'real'.

Firstly, in these tests, the LGMD2 model is tested by a darker object moving in depth within a bright background. As illustrated in Fig. 9, the LGMD2 responds selectively to darker object approaching instead of receding.

Secondly, we look deeper into the model performance against angular looming stimuli where the object (in Fig.

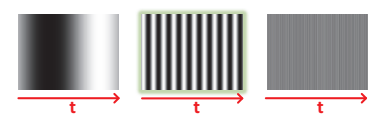

(a) varied grating stimuli

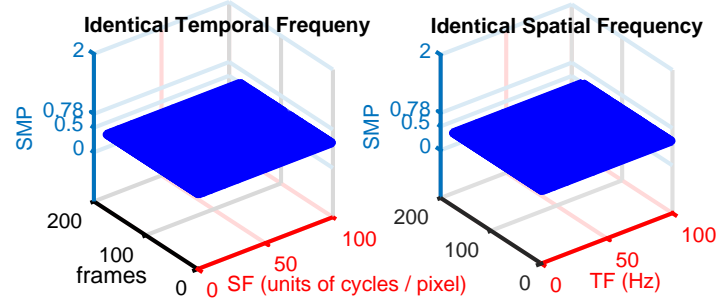

(b) model outputs

Fig. 8. The LGMD2 outputs by sinusoidal gratings with a wide range of spatial (SF) and temporal (TF) frequencies: the firing threshold is set at 0.78.

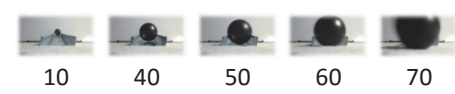

(a) looming stimuli

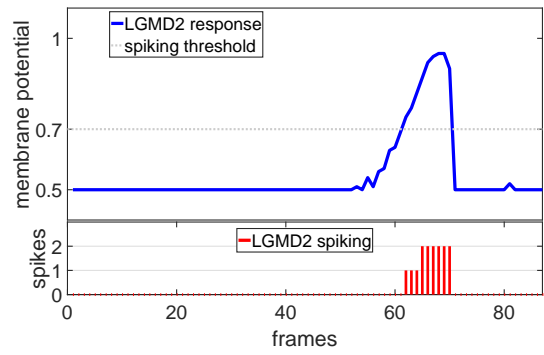

(b) model outputs
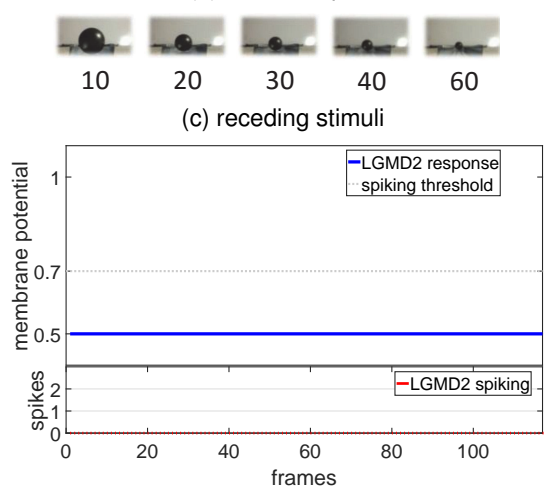

(d) model outputs

Fig. 9. The LGMD2 outputs including potential and spike by a darker object approaching and receding within a bright background in a real physical scene.

9) approaches on with an increased deviation from a direct collision trajectory, as illustrated in Fig. 10a. The statistical results in Fig. 10b demonstrates the LGMD2 spikes at the highest frequency by direct looming stimuli. Along with the approach angle increasing, the LGMD2's output peaks later, and the peak response declines. Though other angular approaches could also activate the neuron, the spike frequency becomes much lower as the object moves away with larger deviation. As introduced in Sections I and II, the locust looming detectors respond most strongly to objects that signal rapid and direct collision threat [15], [37]. The aforementioned OF-based methods and DSNs models can better recognise the angular approaches with different deviations from a direct collision trajectory, since the additional direction information can be extracted by those models. On the other hand, the 


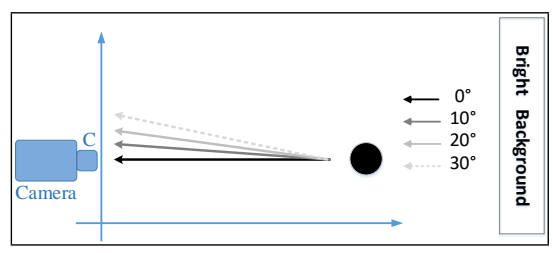

(a) setting the experiments
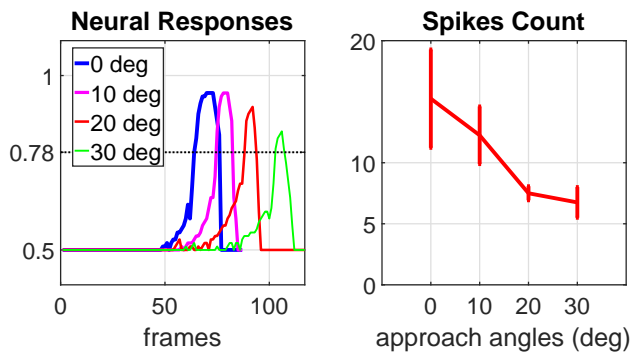

(b) model outputs and statistical results

Fig. 10. Systematic angular-approach testing: (a) setting the experiments, (b) the LGMD2 outputs by a darker object approaching with four distinct angles, and the statistical results of spikes elicited during every course each repeated ten times due to irregular approaching speed and light flash.

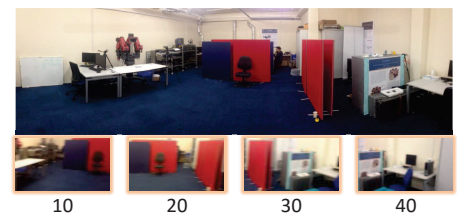

(a) wide-field stimuli

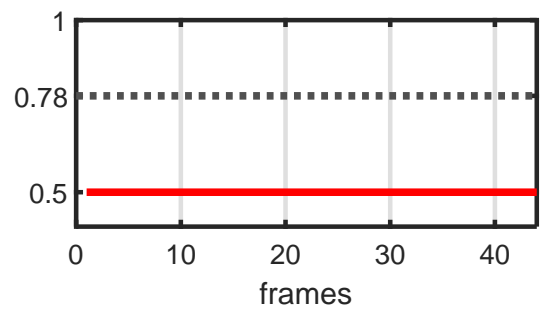

(b) model output

Fig. 11. The LGMD2 model processes a rapid turning cluttered scene.

LGMD2 model cannot tell the directions of translating edges as demonstrated by the translation testing results in Fig. 6, 7 and the biological recordings in Fig. 4.

After that, we also test the proposed model using a rapid turning cluttered indoor scene with transient luminance change over the whole visual field. As shown in Fig. 11, the LGMD2 is rigorously inhibited during the whole-field movement. Similarly to the grating tests in Fig. 8, the proposed adaptive inhibition mechanism works effectively to amplify the lateral inhibitions in the $\mathrm{ON}$ and OFF pathways to suppress the LGMD2 when dealing with such situations.

In the last type of off-line experiments, we investigate the LGMD2's collision detection ability in much more complex and dynamic vehicle driving scenes using recordings from dashboard cameras as the input stimuli. Every scenario contains an urgent crash. Fig. 12 demonstrates the LGMD2 model can well perceive the impending crash representing high spike frequency. Though some isolated spikes could be evoked by non-colliding motions, the LGMD2 spikes at very high rate at the critical moments of threats detected. The results show great potential of the LGMD2 model to build collision-detecting visual systems for ground vehicles.

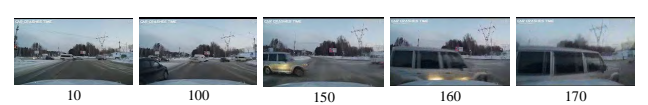

(a) crash video clips

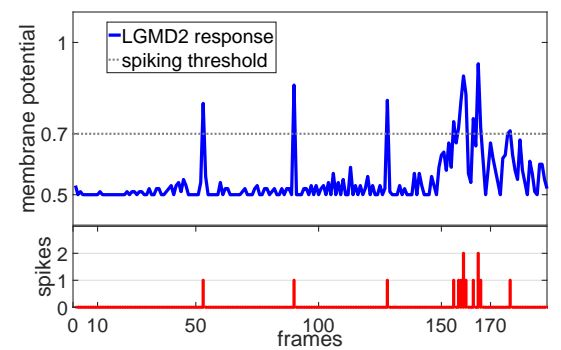

(b) LGMD2 outputs

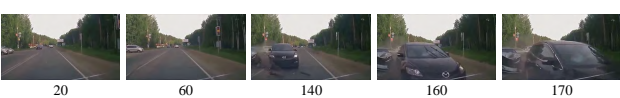

(c) crash video clips

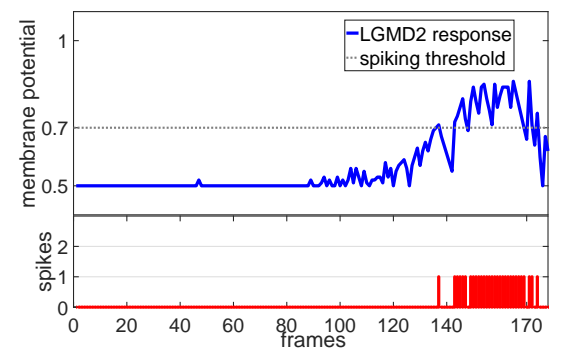

(d) LGMD2 outputs

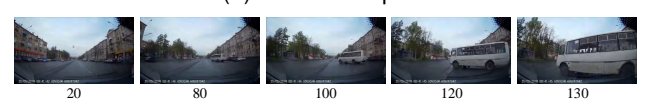

(e) crash video clips

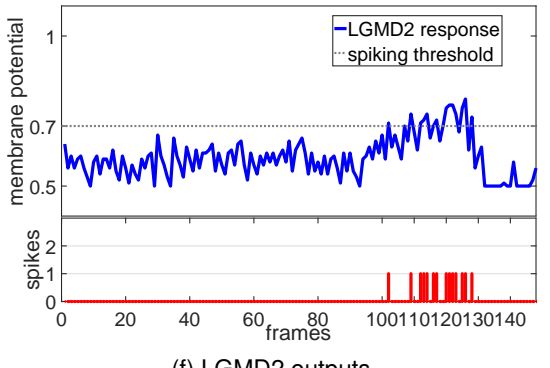

(f) LGMD2 outputs

Fig. 12. The LGMD2 model processes driving scenarios of ground vehicles.

\section{B. On-line Robot Tests}

Within this subsection, the on-line robot tests will be presented. The proposed LGMD2 has been successfully implemented as an embodiment in robot vision. The experiments can be categorised into two types of tests: 1) arena tests: the robot agent is set at different constant linear speeds, and tested in an arena for collision perception and avoidance in near range navigation mixed with many obstacles. 2) bio-robotic tests: the robot agent is tested by overhead approaching stimuli by different grey-scale objects mimicking the situation that a juvenile locust on the ground is stimulated by strikes from predators in the bright sky.

1) Robot Configuration: The mobile robot platform used in the on-line experiments is called 'Colias', as illustrated in Fig. 13. It is a vision-based ground micro robot developed for swarm robotic applications [59]-[61] and bio-robotics research [17], [24], [41]. The Colias robot has two primary boards. The bottom board actuates motion with a maximum speed of $35 \mathrm{~cm} / \mathrm{s}$. The upper board executes vision with a 


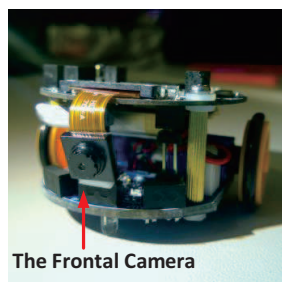

(a)

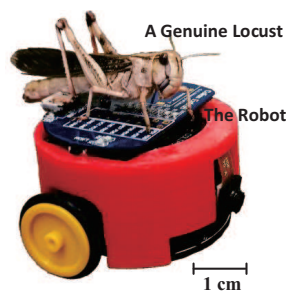

(b)
Fig. 13. Micro mobile robot prototype: (a) the Colias micro-robot used in this research with a monocular camera as the only applied sensor for collision detection, (b) a genuine locust on top of Colias demonstrating its small size.

RGB miniaturised camera, as the only applied sensor in this research, which is capable of operating greater than $30 \mathrm{fps}$. The view angle could reach approximately 70 degrees.

More specifically, for image processing, the Colias robot has limited computation resources. The micro-controller is an ARM Cortex-M4F core, which is deployed as the main processor for monitoring all the modules and serving the task. The $32-$ bit MCU STM32F427 clocked at $180 \mathrm{M} \mathrm{Hz}$ provides the necessary computational power to have a real-time image stream processing. Its 256 Kbytes internal SRAM supports the image buffering and computing.

2) Robot Arena Tests: In the first type of robot experiments, we examine the effectiveness and robustness of the proposed LGMD2 model for guiding the mobile robot for timely collision detection and avoidance in near range navigation in an arena, as depicted in Fig. 14. A Colias robot with the embedded LGMD2 module, as the only collision sensing modality, has been tested in the arena mixed with many obstacles $^{1}$. There are specific patterns on top of the agent and the obstacles for implementing a real-time localisation system [62], in order to get the very precise trajectories of the ID-specific mobile robot and calculate the success rate. In the arena tests, the goalless agent is initialised to go forward autonomously until a potential collision threat detected. The avoidance behaviour is simply set to turn right or left randomly with a large angle, as the mobile robot can only run on the 2D surface. After each avoidance, the agent resumes its forward motion, and so on. Moreover, the constant linear-speed of tested agent is set at 9 levels ranging from extremely slow $(0.1 \mathrm{~cm} / \mathrm{s})$ to the maximum speed $(35 \mathrm{~cm} / \mathrm{s})$.

Fig. 15 illustrates some arena test results with robot overtime trajectories. In general, the robot agent performs consistently and robustly for timely collision perception and avoidance in the arena interacting with obstacles and periphery walls. However, we have noticed the wall issues, with which if the robot gets too close to the wall after turning, it may fail in detecting the collision. In addition, the visual coverage is also limited, with which the robot cannot 'see' the poles in some cases. Table II and Fig. 16 demonstrate statistical success rates (SR) at different tested velocities throughout repeated tests, which can be denoted by

$$
S R=E V_{a} /\left(E V_{a}+E V_{m}\right),
$$

where $E V_{a}$ and $E V_{m}$ indicate the specified events of collision avoidance and miss-detection (hitting the poles or surrounding walls with human intervention during experiments).

\footnotetext{
${ }^{1}$ The videos are with https://github.com/fuqinbing/LGMD2-open-source.
}

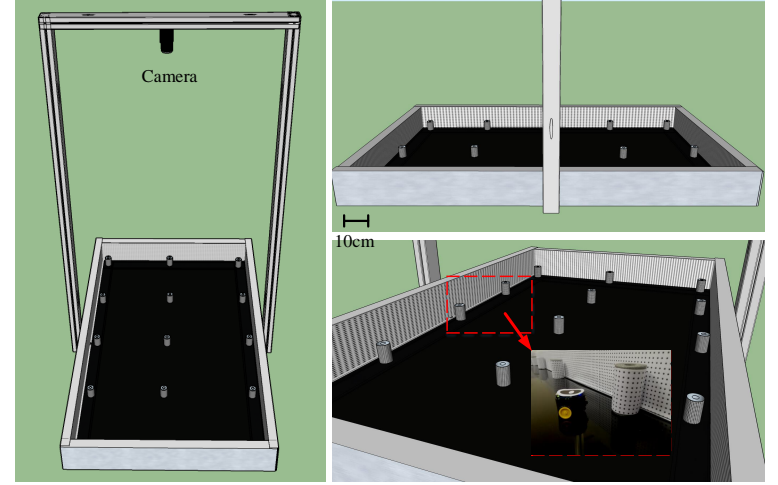

Fig. 14. Schematic illustration of the arena built on a TV screen with the size of 1430 (in length) $\times 805$ (in width) $\times 150$ (in height) $\mathrm{mm}^{3}$. A CCD camera is set on the top of arena to record the robot performance and run a real time localisation system [62]. There are poles as the obstacles in the arena. The walls and surfaces of the poles are decorated with densely distributed dark patterns to stimulate the mobile robot agent.

The arena tests results have verified the flexibility and robustness of the proposed LGMD2 model on the embedded system to guide mobile machines for collision detection in navigation. More concretely, except the extremely slow speed of $0.1 \mathrm{~cm} / \mathrm{s}$, the SR are all above $90 \%$ with satisfactory performance.

3) Bio-robotic Looming Tests: A physiological research has recently revealed that the LGMD2 neuron plays a crucial role in juvenile locusts that mainly live on the ground to recognise proximity of darker targets, e.g. strike from predators in the bright sky [14]. As the first systematic model system to carry out the LGMD2's specific functions, we have also designed bio-robotic tests mimicking the similar scenarios for deepening our understanding of the LGMD2's unique characteristics.

A Colias agent with the LGMD2 visual system is set up in the arena and stimulated by four overhead approaching objects, respectively, each with certain grey scale. The experimental setting is illustrated in Fig. 17. Notably, all objects are darker than their background (the wall of arena): the violet one is the darkest object whilst the yellow one has the smallest contrast to its background. In this kind of experiments, the robot collision avoidance behaviour is configured same to the arena tests. There are also specific patterns on the top of approaching objects for localisation. Therefore, we can track the moving objects, and then obtain the exact positions indicating the activation of robot collision avoidance behaviour.

The following analyses can be drawn from Fig. 18:

1) The LGMD2 agent is able to detect every grey-scale darker object that approaches overhead corresponding to timely evasive move.

2) The LGMD2 model is more sensitive to looming stimuli caused by objects with larger contrast to the background. More precisely, the darker looming objects lead to more frequent avoidance behaviours with relatively longer reaction distances to collision threats; on the other hand, the looming object with the smallest contrast rarely activate the LGMD2 agent with relatively shortest reaction distances to collision threats.

3) The LGMD2 agent responds more constantly to darker objects that approach directly than other angular approaches. The results reconcile with the revealed prop- 

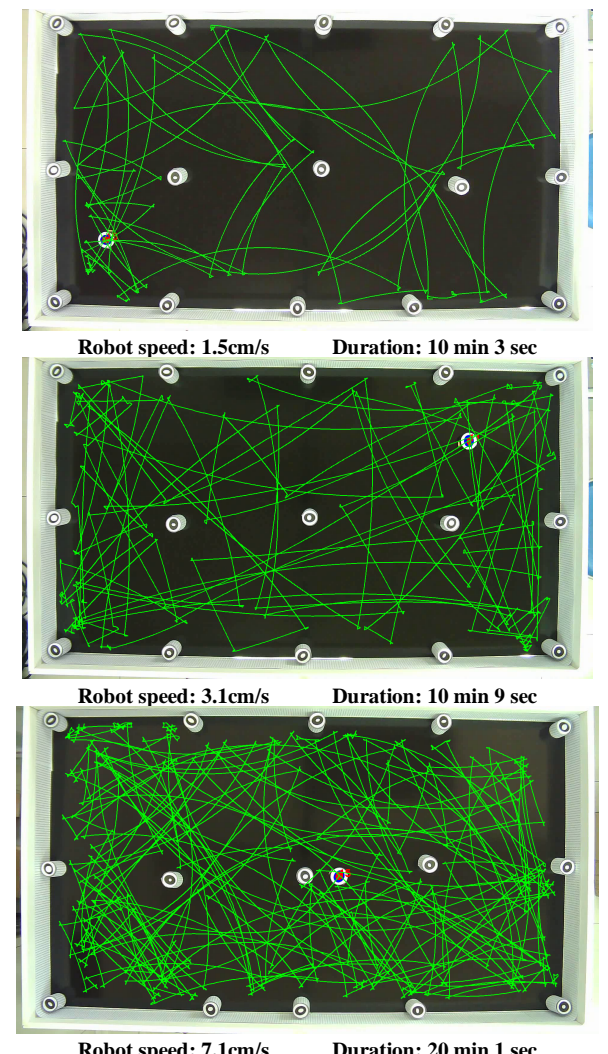

Robot speed: $7.1 \mathrm{~cm} / \mathrm{s}$
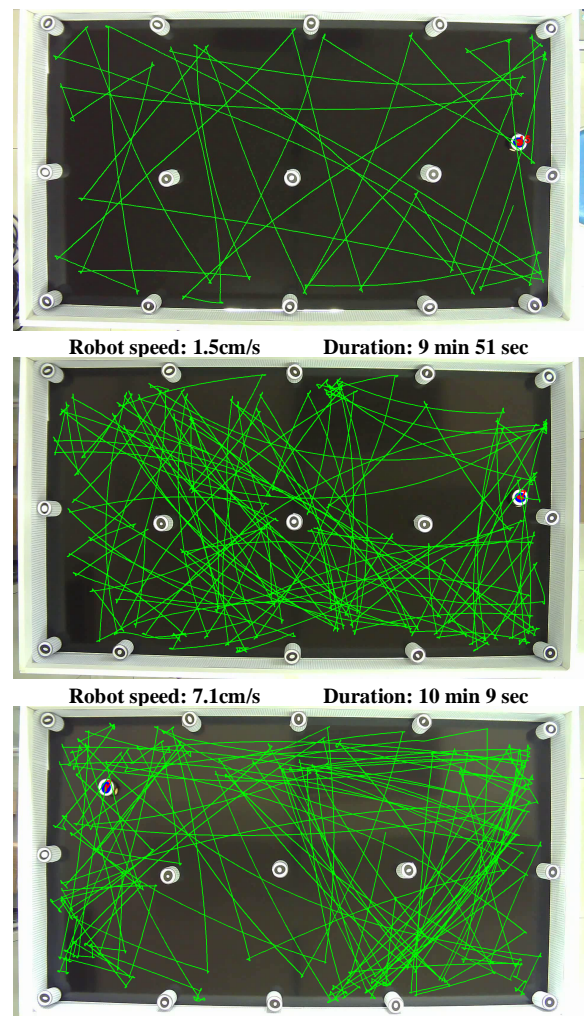

Robot speed: $10.9 \mathrm{~cm} / \mathrm{s}$ Duration: $11 \mathrm{~min} 16 \mathrm{sec}$
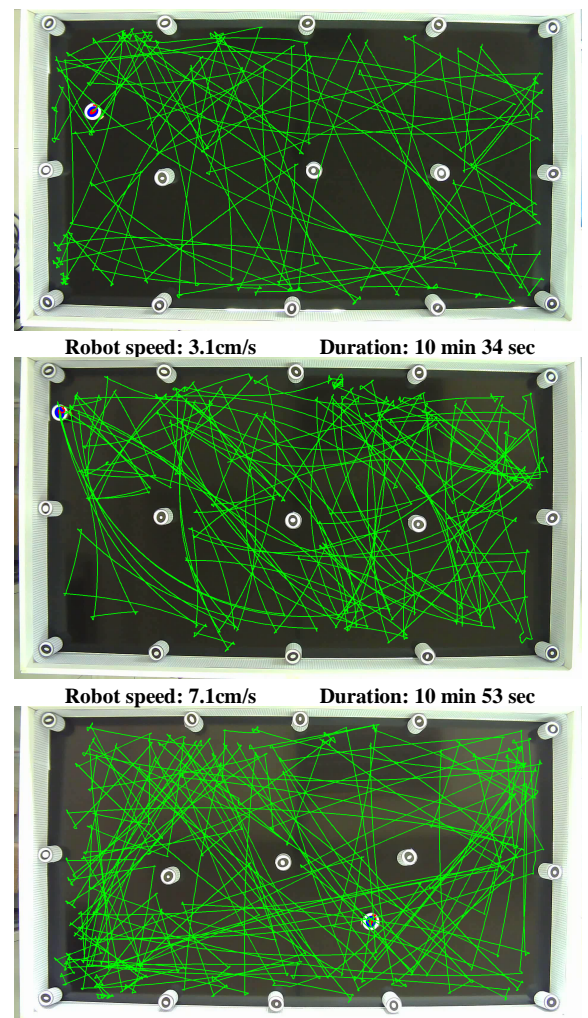

Robot speed: $10.9 \mathrm{~cm} / \mathrm{s}$ Duration: $9 \mathrm{~min} 43 \mathrm{sec}$

Fig. 15. Example results of the Colias robot arena tests for collision avoidance performance represented by trajectories over time (green line). The ID-specific agent has been tested with different constant linear velocities.

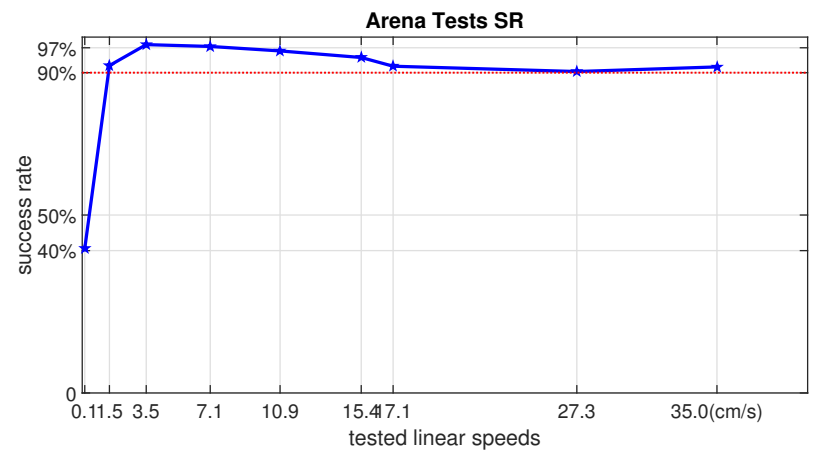

Fig. 16. Success rates of the Colias agent through repeated arena tests at 9 individual linear-speeds for collision perception and avoidance.

erties of locust's LGMDs, which respond most strongly to objects signalling frontal collision threats [15], [17], [37]. This also well matches the angular approach tests results in Fig. 10.

TABLE II

Success Rates of Collision Detection in Arena Tests

\begin{tabular}{|l|l|l|l|}
\hline Speed(cm/s) & Avoidance & Miss-detection & Success rate(\%) \\
\hline 0.1 & 69 & 101 & $40.59 \%$ \\
\hline 1.5 & 506 & 44 & $92.0 \%$ \\
\hline 3.5 & 1250 & 27 & $97.89 \%$ \\
\hline 7.1 & 1703 & 46 & $97.37 \%$ \\
\hline 10.9 & 1755 & 71 & $96.11 \%$ \\
\hline 15.4 & 1841 & 112 & $94.27 \%$ \\
\hline 17.1 & 1436 & 128 & $91.82 \%$ \\
\hline 27.3 & 196 & 21 & $90.32 \%$ \\
\hline 35.0 & 743 & 68 & $91.62 \%$ \\
\hline
\end{tabular}

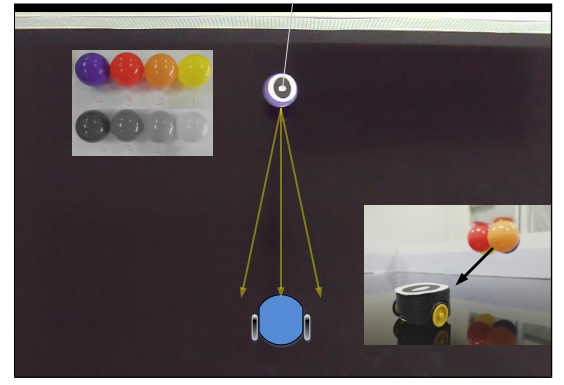

Fig. 17. Setting the bio-robotic looming tests in the arena using objects at four different grey levels as the stimuli. Every grey-scale overhead looming course is repeated a hundred times owing to irregular approaching speed and angle and light flash. The yellow arrows indicate the scope of looming directions including direct and angular approaches.

To sum up, the on-line tests results have demonstrated our achievements on two main aspects: 1) The proposed LGMD2 model has robust performance in the micro mobile robot for collision detection that could be built as low-cost, energyefficient and miniaturised visual sensors for mobile machines. 2) The locust LGMD2's specific selectivity to darker objects has been achieved by the proposed computational modelling.

\section{FURTHER Discussion}

Through the above systematic experiments, we have shown that the proposed LGMD2 visual neural network, with parallel biased-ON and OFF pathways and adaptive lateral inhibitions, demonstrates the similar characteristics and selectivity to biological LGMD2 neurons in the locust's visual systems. In locusts, both the LGMD1 and the LGMD2 respond to rapid expanding image of an approaching object representing an 

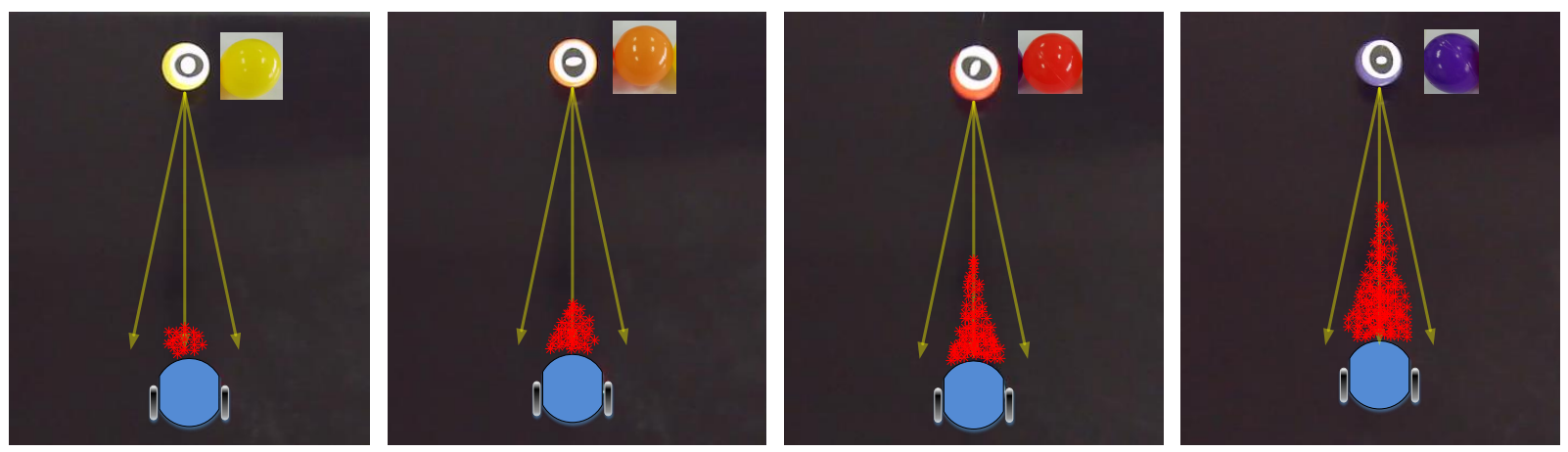

Fig. 18. Illustrations of the locations marked by red asterisks where the avoidance behaviour of the tested robot agent is aroused by overhead looming objects throughout repeated tests under the setting in Fig. 17. The markings of some positions are overlapped.

imminent collision or a strike from predator [11], [12], [15]. Nevertheless, the biological functions of LGMD2 differ from the LGMD1 in a number of ways [12]. First, the LGMD2 is not sensitive to a brighter or white looming object whereas the LGMD1 is. Second, the LGMD2 does not respond to darker objects that recede at all, while the LGMD1 is often excited though very briefly. The proposed computational model has fully exhibited the above two critical features, as shown in Fig. 5 and 9. The model's selectivity to only OFF contrast also satisfies with the biological data in Fig. 4.

The LGMD2 matures earlier than the LGMD1 and plays a crucial role of sensing predators for juvenile locusts living on the ground [14]. As the locusts grow up, the visual environments become more complex due to flying behaviours [63], [64]. The LGMD1 gradually complements the LGMD2 and can deal with other flight related colliding scenarios. A possible reason is that the LGMD1 can recognise also brighter looming objects. However, the LGMD2 still exists in the visual pathways of adult locusts. How both neurons cooperate in the locust's looming perception neural system is still unknown. From a modeller's perspective, a possible way is to build multiple visual pathways combining both the LGMDs to investigate the looming perception in different environments.

It is worth emphasising that the 'darker object' in this study is relative to the brightness of its background. As shown in the experiments, e.g. Fig. 12, a white vehicle is still darker than the bright sky as the background, so the imminent crash is detected; while it is hard to find a brighter object approaching against dark background in real world (see experiments in Fig. S3 of supplementary materials).

There are different methods for shaping the selectivity in such looming sensitive models or neural networks. For example, Badia et al. proposed that the high non-linearity between the feed-forward excitatory and inhibitory responses can well form the selectivity to approaching objects [18]. The FFI pathway in the LGMD1 model, e.g. [11], [41], [51], can also adjust the selectivity at some critical moments such as the end of approach and the start of recession, which cannot be disregarded. In this research, the proposed adaptive inhibition mechanism in the LGMD2 is also an effective approach to make the neuron adaptive to different background motion complexity. Combining with a recent LGMD1 modelling study [17], the bio-plausible structure of ON and OFF pathways could play a role in the locust's visual system to separate the distinct selectivity between the LGMD1 and the LGMD2. In addition, a recent research demonstrates a self-inhibition mechanism could coordinate with the lateral-inhibition to further sharpen up the LGMDs' selectivity to looming versus translation stimuli [15]. In the future, we will investigate these different mechanisms with challenges from a variety of realworld applications.

\section{CONCLUSION}

This article has presented a collision perception visual neural network based on an unique neuron LGMD2 in the locust's visual pathway. The LGMD2 is sensitive to looming objects but only responds selectively to darker objects that approach against bright background underlying a preference for OFF contrast. We have proposed the original computational modelling of biased-ON and OFF pathways with an adaptive inhibition mechanism to fulfil the LGMD2's specific selectivity and characteristics for the perception of darker looming objects. The proposed approach has been validated with systematic experiments ranging from synthetic stimuli tests to real world including vehicle driving scenarios and online robot tests. The experimental results have demonstrated the robustness and flexibility of the proposed LGMD2 visual neural network for collision perception against various dynamic and cluttered backgrounds. The LGMD2 model can be a good candidate visual system to build low-cost and energy-efficient collision-detecting sensors for mobile robots and autonomous vehicles. Similarly to other neuromorphic computation structures, the proposed method can be easily realised in a VLSI chip for volume production.

\section{REFERENCES}

[1] J. Thorson, "Small-signal analysis of a visual reflex in the locust. i. input parameters," Kybernetik, vol. 3, no. 2, pp. 41-53, 1966.

[2] J. S. Kennedy, "The migration of the desert locust (schistocerca gregaria forsk.). i. the behaviour of swarms. ii. a theory of long-range migrations," Philosophical Transactions of the Royal Society of London. Series B, Biological Sciences, vol. 235, pp. 163-290, 1951.

[3] E. Warrant and M. Dacke, "Visual navigation in nocturnal insects," Physiology, vol. 31, no. 3, pp. 182-192, 2016.

[4] E. Baird, E. Kreiss, W. Wcislo, E. Warrant, and M. Dacke, "Nocturnal insects use optic flow for flight control," Biology letters, vol. 7, no. 4, pp. 499-501, 2011.

[5] J. R. Serres and F. Ruffier, "Optic flow-based collision-free strategies: From insects to robots," Arthropod Structure \& Development, vol. 46, no. 5, pp. 703-717, 2017.

[6] Q. Fu, C. Hu, P. Liu, and S. Yue, "Towards computational models of insect motion detectors for robot vision," in Towards autonomous robotic systems conference, 2018, Conference Proceedings, pp. 465-467. 
[7] N. Franceschini, "Small brains, smart machines: From fly vision to robot vision and back again," Proceedings of the IEEE, vol. 102, pp. 751-781, 2014.

[8] Q. Fu, H. Wang, C. Hu, and S. Yue, "Towards computational models and applications of insect visual systems for motion perception: A review," Artificial Life, vol. 25, no. 3, pp. 263-311, 2019.

[9] M. O'Shea and J. L. D. Williams, "The anatomy and output connections of a locust visual interneurone: the lobula giant movement detector (lgmd) neurone," J Comp Physiol, vol. 91, no. 257-266, 1974.

[10] M. O'Shea and C. H. F. Rowell, "The neuronal basis of a sensory analyser, the acridid movement detector system. ii. response decrement, convergence, and the nature of the excitatory afferents to the fan-like dendrites of the lgmd," J Exp Biol, vol. 65, pp. 289-308, 1976.

[11] F. C. Rind and B. D. I., "Neural network based on the input organization of an identified neurone signaling impending collision," J Neurophysiol, vol. 75, pp. 967-985, 1996.

[12] P. J. Simmons and F. C. Rind, "Responses to object approach by a wide field visual neurone, the LGMD2 of the locust: Characterization and image cues," Journal of Comparative Physiology - A Sensory, Neural, and Behavioral Physiology, vol. 180, no. 3, pp. 203-214, 1997.

[13] S. Wernitznig, F. C. Rind, P. Polt, A. Zankel, E. Pritz, D. Kolb, E. Bock, and G. Leitinger, "Synaptic connections of first-stage visual neurons in the locust schistocerca gregaria extend evolution of tetrad synapses back 200 million years," J Comp Neurol, vol. 523, no. 2, pp. 298-312, 2015.

[14] J. Sztarker and F. C. Rind, "A look into the cockpit of the developing locust: looming detectors and predator avoidance," Dev Neurobiol, vol. 74, no. 11, pp. 1078-95, 2014.

[15] F. C. Rind, S. Wernitznig, P. Polt, A. Zankel, D. Gutl, J. Sztarker, and G. Leitinger, "Two identified looming detectors in the locust: ubiquitous lateral connections among their inputs contribute to selective responses to looming objects," Scientific Reports, no. 6, p. 35525, 2016.

[16] S. Yue and F. C. Rind, "A collision detection system for a mobile robot inspired by locust visual system," in IEEE Int. Conf. Robot. Autom., 2005, Conference Proceedings, pp. 3843-3848.

[17] Q. Fu, C. Hu, J. Peng, and S. Yue, "Shaping the collision selectivity in a looming sensitive neuron model with parallel ON and OFF pathways and spike frequency adaptation," Neural Networks, vol. 106, pp. 127-143, 2018.

[18] S. Bermudez i Badia, U. Bernardet, and P. F. Verschure, "Non-linear neuronal responses as an emergent property of afferent networks: a case study of the locust lobula giant movement detector," PLoS Comput Biol, vol. 6, no. 3, p. e1000701, 2010.

[19] P. Cizek, P. Milicka, and J. Faigl, "Neural based obstacle avoidance with CPG controlled hexapod walking robot," in Proceedings of the 2017 IEEE international joint conference on neural networks (IJCNN). IEEE, 2017, Conference Proceedings, pp. 650-656.

[20] L. Salt, G. Indiveri, and Y. Sandamirskaya, "Obstacle avoidance with lgmd neuron: Towards a neuromorphic uav implementation," in Proceedings of the 2017 IEEE international symposium on circuits and systems (ISCAS). IEEE, 2017, Conference Proceedings, pp. 1-4.

[21] J. Zhao, X. Ma, Q. Fu, C. Hu, and S. Yue, "An LGMD based competitive collision avoidance strategy for uav," in Artificial Intelligence Applications and Innovations. Springer International Publishing, 2019, Conference Proceedings, pp. 80-91.

[22] A. Borst and T. Euler, "Seeing things in motion: models, circuits, and mechanisms," Neuron, vol. 71, no. 6, pp. 974-94, 2011.

[23] A. Borst and M. Helmstaedter, "Common circuit design in fly and mammalian motion vision," Nature Neuroscience, vol. 18, no. 8, pp. 1067-1076, 2015.

[24] Q. Fu, S. Yue, and C. Hu, "Bio-inspired collision detector with enhanced selectivity for ground robotic vision system," in British machine vision conference. BMVA Press, 2016, Conference Proceedings, pp. 1-13.

[25] Q. Fu, C. Hu, T. Liu, and S. Yue, "Collision selective LGMDs neuron models research benefits from a vision-based autonomous micro robot," in IEEE International Conference on Intelligent Robots and Systems, 2017, Conference Proceedings, pp. 3996-4002.

[26] Q. Fu and S. Yue, "Modelling LGMD2 visual neuron system," in Proceedings of the 2015 IEEE 25th international workshop on machine learning for signal processing. IEEE, 2015, pp. 1-6.

[27] A. Mukhtar, L. Xia, and T. B. Tang, "Vehicle detection techniques for collision avoidance systems: A review," IEEE Transactions on Intelligent Transportation Systems, vol. 16, no. 5, pp. 2318-2338, 2015.

[28] G. N. DeSouza and A. C. Kak, "Vision for mobile robot navigation: A survey," IEEE Transactions on Pattern Analysis and Machine Intelligence, vol. 24, pp. 237-267, 2002.
[29] F. Poiesi and A. Cavallaro, "Detection of fast incoming objects with a moving camera," in British machine vision conference. BMVA Press, 2016, Conference Proceedings, pp. 1-11.

[30] D. Holz, S. Holzer, R. B. Rusu, and S. Behnke, "Real-time plane segmentation using RGB-D cameras," in RoboCup 2011: robot soccer world cup $X V$. Springer, 2012.

[31] B. Peasley and S. Birchfield, "Real-time obstacle detection and avoidance in the presence of specular surfaces using an active 3D sensor," in Proceedings of the 2013 IEEE workshop on robot vision. IEEE, 2013, Conference Proceedings, pp. 197-202.

[32] H. Kim, S. Leutenegger, and A. J. Davison, "Real-time 3D reconstruction and 6-DoF tracking with an event camera," in European Conference on Computer Vision, 2016, Conference Proceedings, pp. 1-16.

[33] A. R. Vidal, H. Rebecq, T. Horstschaefer, and D. Scaramuzza, "Ultimate SLAM? combining events, images, and imu for robust visual SLAM in HDR and high speed scenarios," IEEE Robotics and Automation Letters, vol. 3, no. 2, pp. 994-1001, 2018.

[34] W. E. Green and P. Y. Oh, "Optic-Flow-Based Collision Avoidance," IEEE Robotics Automation Magazine, vol. 15, no. 1, pp. 96-103, 2008.

[35] J. Serres and F. Ruffier, "Optic flow-based robotics," Wiley Encyclopedia of Electrical and Electronics Engineering, pp. 1-14, 2016.

[36] F. C. Rind and P. J. Simmons, "Seeing what is coming: Building collision sensitive neurons," Trends Neurosci., vol. 22, pp. 215-220, 1999.

[37] J. M. Yakubowski, G. A. Mcmillan, and J. R. Gray, "Background visual motion affects responses of an insect motion-sensitive neuron to objects deviating from a collision course," Physiological Reports, vol. 4, no. 10, p. e12801, 2016.

[38] S. Yue, F. C. Rind, M. S. Keil, J. Cuadri, and R. Stafford, "A bio-inspired visual collision detection mechanism for cars: Optimisation of a model of a locust neuron to a novel environment," Neurocomputing, vol. 69 , no. 13-15, pp. 1591-1598, 2006.

[39] M. Hartbauer, "Simplified bionic solutions: A simple bio-inspired vehicle collision detection system," Bioinspiration \& Biomimetics, vol. 12, no. 2, p. 026007, 2017.

[40] Q. Fu, N. Bellotto, H. Wang, F. C. Rind, H. Wang, and S. Yue, "A visual neural network for robust collision perception in vehicle driving scenarios," in Artificial Intelligence Applications and Innovations. Springer International Publishing, 2019, Conference Proceedings, pp. 67-79.

[41] C. Hu, F. Arvin, C. Xiong, and S. Yue, "Bio-inspired embedded vision system for autonomous micro-robots: The LGMD case," IEEE Transactions on Cognitive and Developmental Systems, vol. 9, no. 3, pp. 241-254, 2017.

[42] H. Meng, K. Appiah, S. Yue, A. Hunter, M. Hobden, N. Priestley, P. Hobden, and C. Pettit, "A modified model for the lobula giant movement detector and its FPGA implementation," Computer Vision and Image Understanding, vol. 114, pp. 1238-1247, 2010.

[43] S. Yue and C. Rind, "Postsynaptic organization of directional selective visual neural networks for collision detection," Neurocomput, vol. 103 , pp. 50-62, 2013.

[44] A. Riehle and N. Franceschini, "Motion detection in flies: Parametric control over on-off pathways," Experimental brain research, vol. 54, no. 2, pp. 390-394, 1984.

[45] J. Antolik, "Rapid long-range disynaptic inhibition explains the formation of cortical orientation maps," Frontiers in Neural Circuits, vol. 11, no. 21, p. 00021, 2017.

[46] T. W. Troyer, A. E. Krukowski, N. J. Priebe, and K. D. Miller, "Contrastinvariant orientation tuning in cat visual cortex: Thalamocortical input tuning and correlation-based intracortical connectivity," The Journal of Neuroscience, vol. 18, no. 15, pp. 5908-5927, 1998.

[47] L. Chariker, R. Shapley, and L.-S. Young, "Orientation selectivity from very sparse lgn inputs in a comprehensive model of macaque v1 cortex," The Journal of Neuroscience, vol. 36, no. 49, pp. 12 368-12 384, 2016

[48] S. D. Wiederman, P. A. Shoemaker, and D. C. O'Carroll, "Correlation between off and on channels underlies dark target selectivity in an insect visual system," J Neurosci, vol. 33, no. 32, pp. 13225-32, 2013.

[49] A. C. James and D. Osorio, "Characterisation of columnar neurons and visual signal processing in the medulla of the locust optic lobe by system identification techniques," Journal of Comparative Physiology A, vol. 178, pp. 183-199, 1996.

[50] M. S. Keil, E. Roca-Moreno, and A. Rodriguez-Vazquez, "A neural model of the locust visual system for detection of object approaches with real-world scenes," in Proceedings of the fourth IASTED international conference on visualization, imaging, and image processing. IASTED, 2004, Conference Proceedings, pp. 340-345.

[51] S. Yue and F. C. Rind, "Collision detection in complex dynamic scenes using a lgmd based visual neural network with feature enhancement," IEEE Trans. Neural Netw., vol. 17, no. 3, pp. 705-716, 2006. 
[52] H. Wang, J. Peng, and S. Yue, "A directionally selective small target motion detecting visual neural network in cluttered backgrounds," IEEE Transactions on Cybernetics, pp. 1-15, 2018.

[53] H. Wang, J. Peng, Q. Fu, H. Wang, and S. Yue, "Visual cue integration for small target motion detection in natural cluttered backgrounds," in Proceedings of the 2019 IEEE International Joint Conference on Neural Networks. IEEE, 2019, Conference Proceedings.

[54] H. Wang, Q. Fu, H. Wang, J. Peng, P. Baxter, C. Hu, and S. Yue, "Angular velocity estimation of image motion mimicking the honeybee tunnel centring behaviour," in Proceedings of the 2019 IEEE International Joint Conference on Neural Networks. IEEE, 2019, Conference Proceedings.

[55] H. Wang, Q. Fu, H. Wang, J. Peng, and S. Yue, "Constant angular velocity regulation for visually guided terrain following," in Artificial Intelligence Applications and Innovations. Springer International Publishing, 2019, Conference Proceedings, pp. 597-608.

[56] Q. Fu and S. Yue, "Modeling direction selective visual neural network with on and off pathways for extracting motion cues from cluttered background," in International Joint Conference on Neural Networks, 2017, Conference Proceedings, pp. 831-838.

[57] Q. Fu, N. Bellotto, C. Hu, and S. Yue, "Performance of a visual fixation model in an autonomous micro robot inspired by drosophila physiology," in Proceedings of the 2018 IEEE International Conference on Robotics and Biomimetics. IEEE, 2018, Conference Proceedings, pp. 1802-1808.

[58] S. Yue and F. C. Rind, "Redundant neural vision systemscompeting for collision recognition roles," IEEE Transactions on Autonomous Mental Development, vol. 5, no. 2, pp. 173-186, 2013.

[59] C. Hu, Q. Fu, T. Liu, and S. Yue, "A hybrid visual-model based robot control strategy for micro ground robots," in From animals to animats 15. Springer International Publishing, 2018, Conference Proceedings, pp. 162-174.

[60] C. Hu, Q. Fu, and S. Yue, "Colias IV: the affordable micro robot platform with bio-inspired vision," in Towards autonomous robotic systems conference. Springer International Publishing, 2018, Conference Proceedings, pp. 197-208.

[61] X. Sun, T. Liu, C. Hu, Q. Fu, and S. Yue, "ColCOS $\phi$ : A multiple pheromone communication system for swarm robotics and social insects research," in Proceedings of the 2019 IEEE 4th International Conference on Advanced Robotics and Mechatronics (ICARM). IEEE, 2019, Conference Proceedings, pp. 59-66.

[62] T. Krajník, M. Nitsche, I. Faigl, P. Vaněk, M. Saska, L. Přeučil, T. Duckett, and M. Marta, "A practical multirobot localization system," Journal of Intelligent and Robotic Systems, vol. 76, no. 3-4, pp. 539$562,2014$.

[63] P. J. Simmons, J. Sztarker, and F. C. Rind, "Looming detection by identified visual interneurons during larval development of the locust locusta migratoria," J Exp Biol, vol. 216, no. Pt 12, pp. 2266-75, 2013.

[64] P. J. Simmons, F. C. Rind, and R. D. Santer, "Escapes with and without preparation: the neuroethology of visual startle in locusts," J Insect Physiol, vol. 56, no. 8, pp. 876-83, 2010.

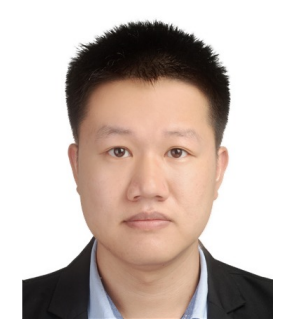

Cheng $\mathrm{Hu}$ received the B.Eng. degree from the College of Opt-Electronics Engineering, Chongqing University, Chongqing, China in 2013, the Ph.D. degree from University of Lincoln, Lincoln, UK in 2018. He was a recipient of the Marie Curie Fellowship to be involved in the EU FP7 projects EYE2E(269118) and LIVCODE(295151) and EU Horizon 2020 projects STEP2DYNA(691154) and ULTRACEPT(778062). He is now a Honorary Postdoctoral Research Fellow in University of Lincoln, a Postdoctoral Research Fellow in Guangzhou University. His current research interests include bio-inspired algorithms, biorobotics, autonomous robots, sensors, and signal processing.

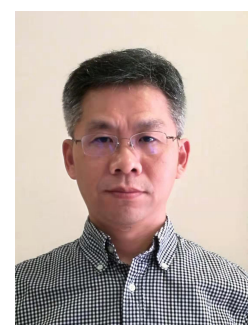

Jigen Peng received the B.Sc. degree from Jiangxi University, Nanchang, China, in 1989, the M.Sc. and the Ph.D. degrees from Xi'an Jiaotong University, Xi' an, China, in 1992 and 1998, respectively. He is currently a Professor with the School of Mathematics and Information Science, Guangzhou University, Guangzhou, China. He worked in Xi' an Jiaotong University and City University of Hong Kong before 2018. His current research interests include nonlinear functional analysis and applications, machine learning theory, and sparse information processing.

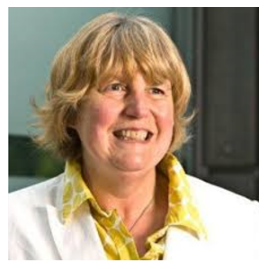

F. Claire Rind received the B.Sc. degree in animal physiology from the University of Canterbury, Christchurch, New Zealand, in 1976, and the Ph.D. in zoology from Cambridge University, Cambridge, U.K., in 1982.

She is currently a Reader in Invertebrate Neuroscience in the School of Biology and at the Institute of Neuroscience, Newcastle University, Newcastle, U.K. Previously, she held a Royal Society University Research Fellowship and Biotechnology and Biological Sciences Research Council (BBSRC) Advanced Research Fellowship. Her current research interests include sensory processing by the insect brain, neuronal pathways for collision avoidance in locusts, bioinspired robotics and Application Specific Integrated Circuits (ASICS) for visual tasks. Dr. Rind is a Member of the Society for Experimental Biology, the Physiological Society, and the International Society for Neuroethology.

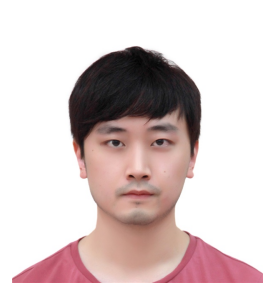

Qinbing Fu (M'18) received the B.Sc. degree from the University of Electronic Science and Technology of China in 2009, the M.Sc. by Research degree from the University of Lincoln in 2014, the Ph.D. degree from the University of Lincoln in 2018.

He was awarded the Marie Curie Fellowship to be involved in the EU FP7 projects EYE2E(269118) and LIVCODE(295151), EU Horizon 2020 projects STEP2DYNA(691154) and ULTRACEPT(778062) as a Research Assistant with the School of Mathematics and Statistics, Xi' an Jiaotong University, Xi' an, China, and the Institute of Microelectronics, Tsinghua University, Beijing, China, from 2014 to 2018. He is now a Honorary Postdoctoral Research Fellow in the University of Lincoln, a Postdoctoral Research Fellow in the Guangzhou University. His current research interests include computer vision, bio-inspired algorithms, bio-robotics and machine learning. Dr. Fu is a Member of IEEE, Robotics and Automation Society (RAS) and International Neural Network Society (INNS).
Shigang Yue (M'05-SM'17) received the M.Sc. and Ph.D. degrees from the Beijing University of Technology (BJUT), Beijing, China, in 1993 and1996, respectively.

He was with BJUT as a Lecturer from 1996 to 1998 and an Associate Professor from 1998 to 1999. He was an Alexander von Humboldt Research Fellow with the University of Kaiserslautern, Kaiserslautern, Germany, from 2000 to 2001. He held research positions at the University of Cambridge, Cambridge, U.K., Newcastle University, Newcastle upon Tyne, U.K., and University College London (UCL), London, U.K, during 2002-2007. In 2007, he joined the University of Lincoln, Lincoln, U.K., as a Senior Lecturer and was promoted to Professor in 2012, where he is currently a Professor (part-time) with the School of Computer Science. He also holds a Professorship with the Machine Life and Intelligence Research Centre, Guangzhou University, Guangzhou, China, in collaboration with Prof. J. Peng. His current research interests include artificial intelligence, computer vision, robotics, intelligent transportation, brains, and neuroscience. He is a senior member of IEEE, a member of International Neural Network Society (INNS) and International Society of Bionic Engineering (ISBE). 


\title{
Supplementary Materials
}

\author{
Qinbing Fu, Member, IEEE, Cheng Hu, Jigen Peng, F. Claire Rind and Shigang Yue, Senior Member, IEEE
}

We have presented a visual neural network inspired by the LGMD2 giant neuron in the locust's visual systems for robust perception of darker objects that approach within bright background. We have evaluated the performance of the proposed model, systematically, with stimuli including synthetic and real-world scenarios and compared the realised specific selectivity to previous physiological data on the locust LGMD2. We have also implemented the proposed model on the embedded system in a micro mobile robot for guiding collision detection and avoidance in near range navigation. For the first time, the locust LGMD2 neuron has been studied, systematically, through computational modelling and experimenting. To further demonstrate the LGMD2's unique characteristics, we have also tested the LGMD2 visual system with two types of systematic experiments.

The supplementary materials are organised as follows: In Section S.I, the model is tested with recorded translations in off-line experiments. In Section S.II, we introduce two sets of bio-robotic tests in on-line experiments.

\section{S.I. OfF-Line Translation TESTING}

The locust LGMD2 responds most strongly to approaching darker objects over other categories of movements including translations. To verify whether our proposed neuron model possesses the similar character, the visual system is challenged by a few sets of translations against a visually cluttered background, as shown in Fig. S1a. The statistical results in Fig. S1b demonstrate that 1) the proposed LGMD2 model exhibits lower-level peak response compared to the looming tests, and more importantly, the peaks are all below the defined firing threshold; 2) the proposed LGMD2 model represents speed response to the translating stimuli with different speed levels, that is, the faster translating stimuli bring about stronger response of the proposed model.

\section{S.II. ON-LINE BIO-ROBOtIC TESTING}

We also investigate the underlying characteristics of the proposed model on the embedded system. In this kind of online experiments, the motion unit of the Colias robot with the LGMD2 visual system is closed. We collect the model outputs

This research has been supported by the EU Horizon 2020 projects STEP2DYNA (691154) and ULTRACEPT (778062). Correspondence: Shigang Yue (syue@lincoln.ac.uk), Qinbing Fu (qifu@lincoln.ac.uk).

Q. Fu, C. Hu and S. Yue are with the Machine Life and Intelligence Research Centre, School of Mechanical and Electrical Engineering, Guangzhou University, Guangzhou 510006, China, and also with the Computational Intelligence Lab, Lincoln Centre for Autonomous Systems, School of Computer Science, University of Lincoln, Lincoln LN6 7TS, United Kingdom.

J. Peng is with the School of Mathematics and Information Science, Guangzhou University, Guangzhou 510006, China.

F. C. Rind is with the Institute of Neuroscience/Centre for Behaviour and Evolution, Newcastle University, Newcastle NE1 7RU, United Kingdom.

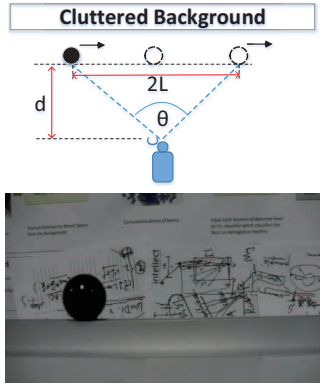

(a) setting the experiments

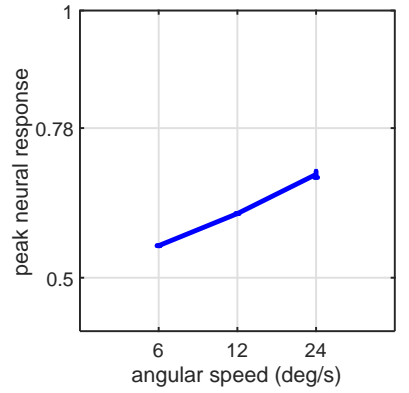

(b) statistical results
Fig. S1. Systematic translation testing: (a) Setting the experiments with an example snapshot of translating object from video clips: the distance $d$ is fixed, and the angular size can be denoted by $\theta=2 \tan ^{-1}(L / 2 d)$ for estimating the angular speed of translating object. (b) The statistics of peak outputs of the proposed model tested by translations at three angular speeds, each throughout ten repeated courses due to irregular speed and light flash.

using Bluetooth. The experimental setting is illustrated in Fig. S2. Notably, with respect to the biological experiments on the locust LGMD2, we set up a bright and a dark environments, respectively. We use five different colour objects, from white to dark, to form overhead looming stimuli to the robot agent. Each RGB-colour object possesses a certain grey level.

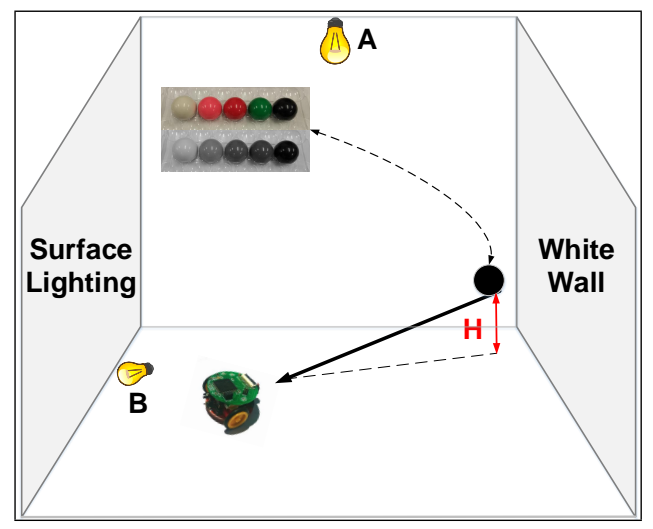

Fig. S2. Setting the overhead looming tests: five grey-scale objects approach the Colias agent along a slot with a fixed slope to the ground (height $H$ ), respectively. There are two light sources capable of forming either a light or a dark environment. The lighting point A alone is a global illumination to form a bright environment. And the lighting source B on its own supports a local surface illumination behind the robot to form a dark environment.

In the first case, only the global illumination (source A in Fig. S2) is applied to make up a purely bright background. As a result, all objects including the white one are darker than the background (Fig. S3a). Each looming stimulus leads to OFF contrast (the light-to-dark luminance change). It can be seen from Fig. S3b that the LGMD2's membrane potential steeply increases by darker objects approaching. The LGMD2 model 


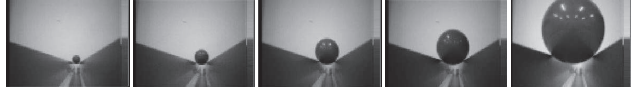

(a) sampled views of darker loom

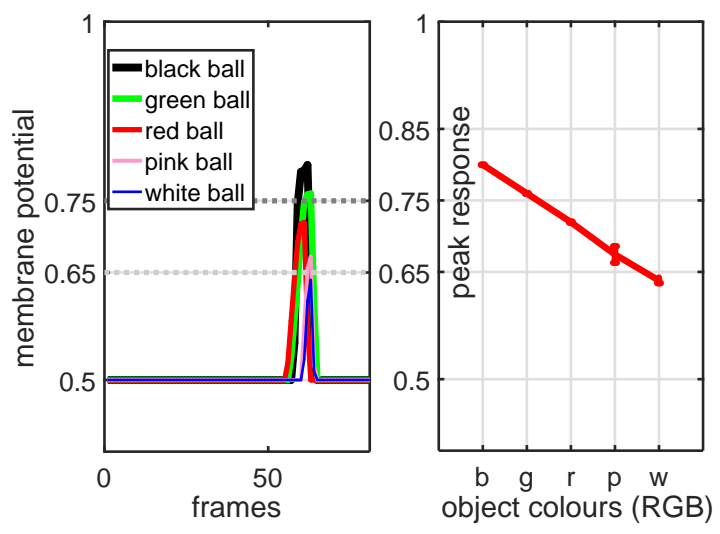

(b) results of darker looming tests

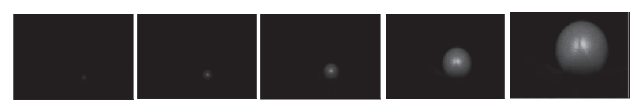

(c) sampled views of brighter loom
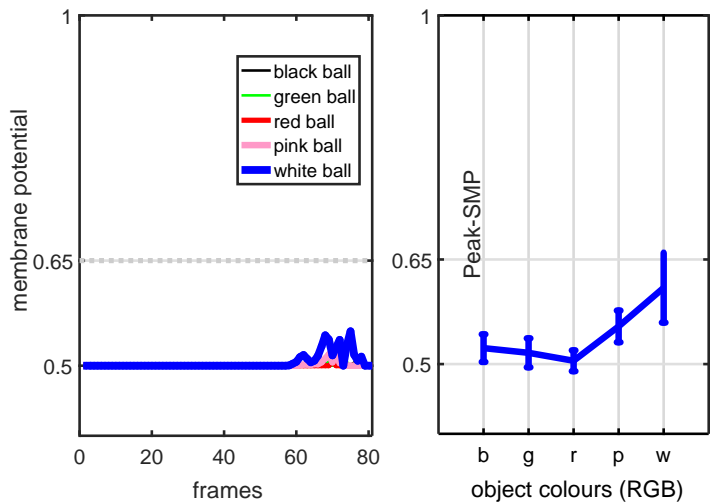

(d) results of brighter looming tests

Fig. S3. The results of overhead looming tests under the setting in Fig. S2: the example views adapted from the tested robot agent are shown in (a), (c). Each grey-scale looming course is repeated ten times in bright and dark environments, respectively, due to irregular approaching speed and light flash. Horizontal dashed lines indicate spiking threshold levels.

can well perceive collision threats by most of the grey-scale approaching stimuli, yet the white looming object gives rise to a relatively weaker response, which could not activate the LGMD2 sufficiently for producing high-frequency spikes (Fig. S3b). More intuitively, the statistical results from repeated tests reveal that the proposed LGMD2 model performs consistently on perceiving darker looming objects with varied grey levels. In addition, it appears that the approaching objects with larger contrast bring about stronger model response, which means the proposed LGMD2 is also sensitive to the contrast between the looming object and its background.

On the other hand, we change the illumination by replacing the global lighting with the local surface one (B in Fig. S2). In this case, as shown in Fig. S3c, all targets including the darkest object are lighter than the background. Therefore, each looming course brings about ON contrast (the darkto-light luminance change). Fig. S3d demonstrates that the
LGMD2 model cannot detect these collisions by brighter approaching objects. Although the white looming object leads to the strongest response, the peak responses are all below the defined firing threshold (Fig. S3d). This is exactly consistent with the revealed neural properties of biological LGMD2 in locusts that are sensitive to looming objects with OFF contrast rather than $\mathrm{ON}$ contrast. 